\title{
Reise eines deutschen Professors ins Eldorado.
}

\author{
von Ludwig Boltzmann
}

1905

Nach der Ausgabe der Populären Schriften, 1905 neu herausgegegeben und mit einem Personenregister versehen von Gabriele Dörflinger, Universitätsbibliothek Heidelberg, 2005.

Heidelberger Texte zur Mathematikgeschichte

\section{Inhaltsverzeichnis}

Reise eines deutschen Professors ins Eldorado 


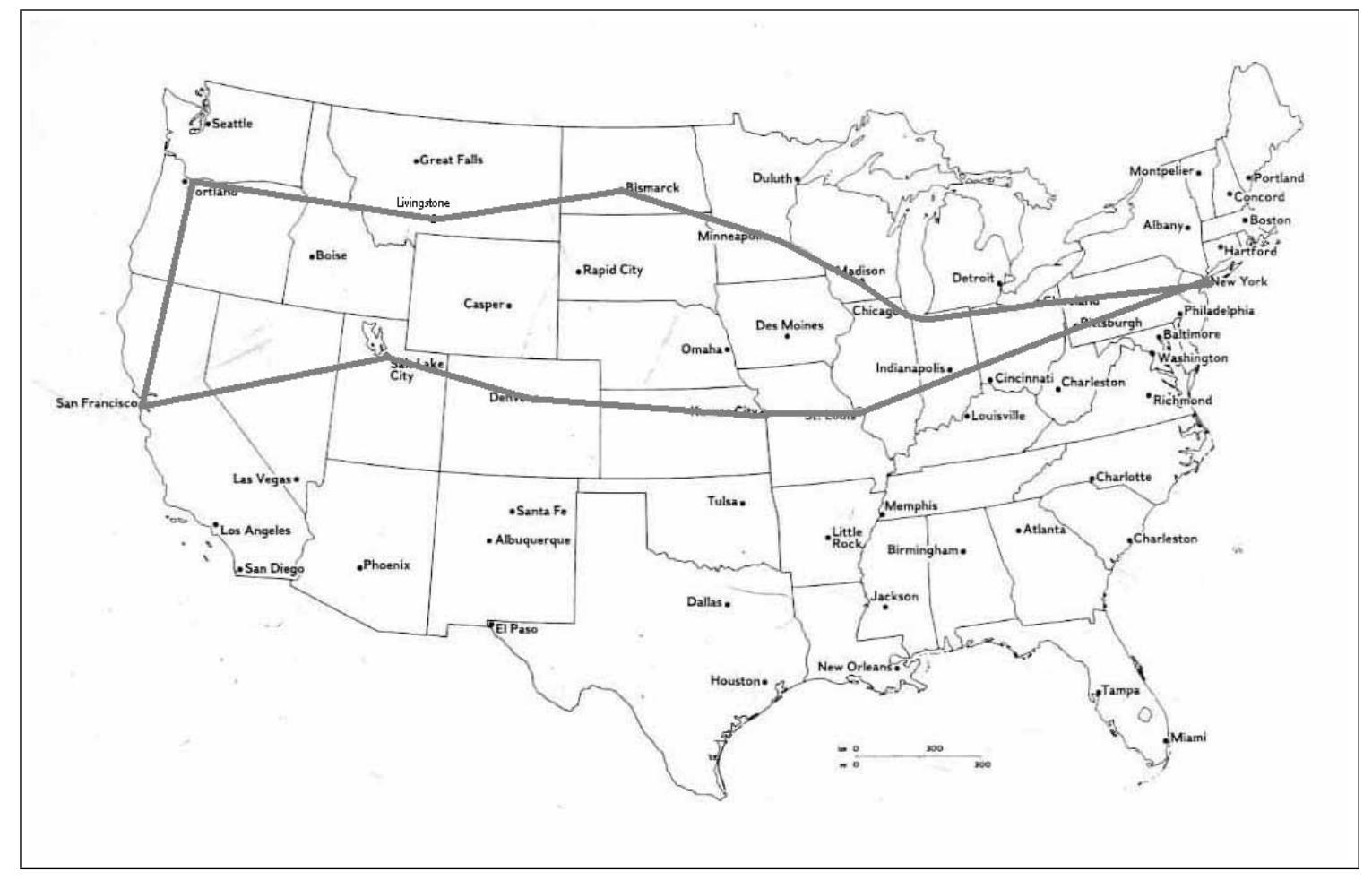

Reiseroute Boltzmanns in Amerika

Die Route der Hinfahrt von New York über St. Louis ist nicht gesichert. Boltzmann hätte auch über Chicago und Omaha nach San Francisco fahren können.

Zu der „Summer School“ 1905 in Berkeley vgl.

Allen, Albert H.: University record

In: The University chronicle / University of California, 7,1904-05. - S. 323326.

UB-Signatur: F 2710-5::7.1904-05 


\section{Reise eines deutschen Professors ins Eldorado}

Da ich schon mehrmals in Amerika, einmal in Konstantinopel, Athen, Smyrna und Algier war, so fehlten mir auch nicht Aufforderungen, einige von meinen Reiseerlebnissen drucken zu lassen. Mir erschien alles doch zu unbedeutend; aber meine letzte Reise nach Kalifornien war schon eher etwas Exquisites und so soll denn eine kleine Plauderei darüber gewagt sein.

Ich will beileibe nicht etwa sagen, daß man durchaus nach Kalifornien reisen müßte, um Interessantes und Schönes zu sehen und sich zu freuen. Man kann auch bei einer Tour in den schönen Bergen unseres Vaterlandes so viel Lust und Freude empfinden als in einer Menschenbrust Platz hat. Man kann bei ganz einfachem Mahle so vergnügt sein wie ein König, aber eine Reise nach Kalifornien ist Champagner veuve Clicquot und Austern.

Der erste Teil meiner Reise stand unter dem Zeichen der Eile und in Eile soll er auch erzählt sein. Noch am 8. Juni wohnte ich der Donnerstagssitzung der Wiener Akademie der Wissenschaften in gewohnter Weise an. Beim Fortgehen bemerkte ein Kollege, daß ich nicht wie sonst nach der Bäckerstraße, sondern nach dem Stubenring mich wandte und fragte, wohin ich gehe. Nach San Franzisko antwortete ich lakonisch.

Im Restaurant des Nordwestbahnhofes verzehrte ich noch in aller Gemütlichkeit Jungsschweinsbraten mit Kraut und Erdäpfeln und trank einige Gläser Bier dazu. Mein Zahlengedächtnis, sonst erträglich fix, behält die Zahl der Biergläser stets schlecht.

Kein einigermaßen Reisekundiger wird sich wundern, daß ich über das Essen und Trinken spreche. Es ist nicht nur kein unwesentlicher Faktor, es ist vielmehr der Angelpunkt. Das wichtigste auf der Reise ist, den Körper der ganzen Mannigfaltigkeit ungewohnter Einflüsse gegenüber gesund zu erhalten und vor allem den Magen, gar den verwöhnten Wienermagen. Kein Wiener wird ungerührt das letzte Gollasch mit Nockerl essen und konzentriert der Schweizer sein Heimweh in der Erinnerung an den Kuhreihen und die Herdenglocken, so der Wiener an das Geselchte mit Knödel. „Sagt nicht, das Alter machte mich kindisch, es fand mich eben noch als ein wahres Kind."

Als ich mit der Mahlzeit fertig war, kamen meine Frau und meine Kinder mit dem schon vorgerichteten Reisegepäck. Adieu noch und fort ging es, zunächst zu den Akademie-Kartellsitzungen nach Leipzig, welche am nächsten Tag 10 Uhr vormittags begannen. Ich machte mich im Zug noch möglichst rein (da hätte ich die washing rooms der amerikanischen Bahnen brauchen können!), setzte mich nach Ankunft des Zuges in Leipzig sofort in eine Droschke und kam pünktlich zur Sitzung.

Am Tor der Aula traf ich gerade mit Kollegen Credner zusammen, der auch zur Sitzung ging und mir in liebenswürdigster Weise behilflich war, 
meine Gepäckstücke, die ich nirgends hatte absetzen können, in den Vorsaal des Sitzungszimmers hinaufzutragen.

Ich ging zu diesen Kartellsitzungen nicht ohne Angst; denn es sollte ein Gegenstand zur Sprache kommen, der für mich sehr bitter werden konnte.

Wird es den Leser langweilen, wenn ich ihn kurze Zeit in einer Werkstätte wissenschaftlicher Arbeit herumführe, um ihm die äußere Einrichtung zu zeigen und den Mechanismus etwas zu erklären; ich hoffe nicht. Heutzutage gibt es doch kaum einen Gebildeten, der nicht irgend eine größere, im Baedeker angeführte Eisenwaren- oder Leder- oder Glasfabrik gesehen hätte und ich finde die Befriedigung der Neugierde, wie die Gegenstände unseres täglichen Gebrauches in ihre uns so geläufige Form gebracht werden, ebenso unterhaltend als lehrreich. Warum sollte ich nicht auch einige Neugierde nach dem Mechanismus einer Fabrik voraussetzen, die, ich darf es wohl sagen, für die menschliche Kultur wichtiger ist, als die größte Lederfabrik, hoffentlich nicht lederner.

Mehrere deutsche Akademien und gelehrte Gesellschaften haben sich zusammengetan, um jährlich gemeinsame Sitzungen zu halten und dort Gegenstände von allgemeiner Wichtigkeit zu besprechen. Dies ist das AkademieKartell. Dasselbe beschloß vor Jahren die materielle Unterstützung eines großen Buchwerkes, der Enzyklopädie der mathematischen Wissenschaften. Die Mathematik hat nämlich im vorigen Jahrhundert enorm an Umfang zugenommen; dabei hat jeder Autor seine besonderen Bezeichnungen und schreibt oft so schwer verständlich, daß nur die nächsten Fachgenossen mit größter Anstrengung folgen können. Doch ist in dieser schwer verständlichen, oft fast unauffindbaren, in der ganzen Welt zerstreuten mathematischen Literatur ungemein viel des Brauchbaren, auch für den Praktiker Nützlichen, ja fast Unentbehrlichen, vergraben.

Die wohlgeordnete Sammlung und möglichst leicht verständliche Darstellung alles dieses Materials ist nun die Aufgabe der besprochenen Enzyklopädie. Sie soll alles in der Mathematik geleistete für den Mathematiker leicht auffindbar machen und zugleich die Brücke zur Praxis bauen, also dem Praktiker die Mathematik, dem Mathematiker die Praxis näherrücken. Das Bedürfnis nach einer solchen enzyklopädischen Zusammenfassung der mathematischen Literatur springt so in die Augen, daß Professor Klein in Göttingen sie als mathematische Bedürfnisanstalt bezeichnet hat.

Ein solches Unternehmen wäre nicht so enorm schwierig, wenn es sich nur darum handelte, die hervorstechendsten Leistungen ohne allzu genaue Kritik anzuführen und das Allerwichtigste, natürlich aber auch Allerbekannteste, zu registrieren. Wenn man aber auf allen Gebieten alles wirklich Nützliche aus dem Verborgenen hervorziehen, alles Unwesentliche ausscheiden, möglichste Vollständigkeit in der Literaturangabe erzielen und dabei alles in übersichtli- 
cher, für den Gebrauch des Lesers bequemer Form darstellen will, so erscheinen die Schwierigkeiten für jeden, der nur einigermaßen in die mathematische Literatur hineingeguckt hat, fast erschreckend. Den schon genannten Professor Klein lockte dies an, die Akademien geben Geld für die Druckkosten, die Autorenhonorare und Reisediäten, Klein und sein wissenschaftlicher Stab besorgen die Arbeit.

Da gilt es für jedes Spezialgebiet unter allen Nationen des Erdballes denjenigen herauszufinden, der es am besten beherrscht. In der Tat arbeiten Deutsche und Franzosen, Russen und Japaner in Eintracht mit. Der Ausgewählte ist nun oft ein großer Herr, der genug Geld und wenig Zeit, vielleicht auch nicht allzuviel Arbeitslust, aber desto mehr Eigensinn hat. Er muß erstens bewogen werden, daß er einen Beitrag verspricht; dann belehrt und mit allen Mitteln der Überredungskunst dazu vermocht werden, daß er den Beitrag so abfaßt, wie er in den Rahmen des Ganzen paßt und last not least, daß er sein Versprechen auch rechtzeitig hält.

Die Beratungen, ob man einen Artikel, der sich besser später einreihen würde, schon jetzt bringen soll, weil man ihn eben schon hat und die, welche man vorausgehen lassen wollte, noch fehlen, nehmen Stunden in Anspruch. Reisen Kleins selbst und seiner Apostel nach allen Ländern der Welt werden nicht gespart, um den Artikelschuldigen mit der Wucht der persönlichen Rücksprache nicht zu verschonen. Eine Lücke blieb lange offen, weil der dafür Erkorene, ein mathematisch gebildeter russischer Offizier in Port Arthur eingeschlossen war. Ich habe solche Enzyklopädiesitzungen schon oft mitgemacht, von ihrer dramatischen Bewegtheit könnten die deutschen Bühnendichter profitieren.

Nun zu mir zurück. Schon als mir Klein einen Enzyklopädieartikel auftrug, weigerte ich mich lange. Endlich schrieb er mir: „Wenn Sie ihn nicht machen, übergebe ich ihn dem Zermelo". Dieser vertritt gerade die der meinen diametral entgegengesetzte Ansicht. Die sollte doch nicht in der Enzyklopädie die tonangebende werden, daher antwortete ich umgehend: „Ehe der Pestalutz es macht, mache ichs." (Sämtliche Zitate, meist aus Schiller zur Nachfeier des Schillerjahres, sind mit Anführungszeichen versehen; man weise sie nach!)

Jetzt aber ist die Zeit, wo mein Artikel fällig wird. Ich hätte gern mich im September von den Reisestrapazen auf dem Lande erholt, aber ich habe mein Wort gegeben, muß also im September in der Literatur wühlen und mit einer kleinen Kohorte Wiener Physiker zusammen, den Artikel fertigstellen. „Ewigkeit geschworenen Eiden."

Ähnlich scheint es auch Professor Wirtinger ergangen zu sein; denn als Emblem der Enzyklopädie zeichnete er eine Mausefalle; der Speck lockt und der Professor ist gefangen. 
Was aber reizt zu dem ganzen Werke so unwiderstehlich? Besonderer Ruhm ist dabei nicht zu holen, mit Ausnahme dessen, etwas Nützliches geleistet zu haben; vom Gelde rede ich gar nicht. Was veranlaßt Klein mit einer psychologischen Kenntnis, um die ihn die Philosophen beneiden könnten, bei jedem, den er auf dem Korn hat, gerade den wunden Punkt zu treffen, wo er überredungsfähig ist? Doch nur der Idealismus, und tun wir die Augen auf, Idealismus finden wir überall bis an das stille Meer. Dort grüßen uns zwei weiße dicke Türme, die Licksternwarte, das Werk eines Idealisten und hundertfachen Millionärs; später mehr davon. Ich habe lange überlegt, was merkwürdiger ist, daß in Amerika Millionäre Idealisten, oder daß Idealisten Millionäre sind. Glücklich das Land, wo Millionäre ideal denken und Idealisten Millionäre werden! In allen Ehren dabei das Selchfleisch mit Knödel; Idealisten brauchen überall einen guten Magen.

Der Idealismus Kleins und seiner Mitarbeiter trug gute Früchte. Schon nach dem Erscheinen der ersten Hefte mußte die Auflage vermehrt werden; eine französische Übersetzung ist angefangen, eine englische wird bald folgen. Die Akademien haben einen guten Griff und der Buchhändler hat ein gutes Geschäft gemacht.

Die Berliner Akademie der Wissenschaften gehört leider dem Kartell nicht an und beteiligt sich gar nicht an der Sache. Sie war auch auf dem Meteorologenkongreß zu Southport und auf dem Sonnenforschungskongresse in St. Louis gar nicht vertreten. Ich fürchte, durch dieses Prinzip, an allem, was sie nicht selbst angefangen hat, sich nicht zu beteiligen, wird sie mehr noch als die Wissenschaft, sich selbst und Deutschland schädigen. Mich ärgerte es, als in Southport und St. Louis unter den foreigners (Nichtengländern) überall die Franzosen den ersten Platz erhielten. Wir Deutsche hätten es wahrlich nicht nötig, ihnen nachzustehen! Aber was vermochte ich als Österreicher? Wenn bei den Meteorologen noch Hann da gewesen wäre, den alle so vermißten! Aber der ist wieder nicht zum Reisen zu bewegen!

Wenn ich schon ins Plaudern komme, dann lasse ich meiner Zunge völlig freien Lauf. So verschweige ich auch nicht, daß ein amerikanischer Kollege überhaupt von einem Rückgang Berlins sprach. In der Tat gingen unter Weierstraß, Kronecker, Kummer, Helmholtz, Kirchhoff die amerikanischen Mathematiker und Physiker meist nach Berlin studieren, jetzt bevorzugen sie Cambridge und Paris. Dadurch, daß es weniger mehr von den Deutschen lernt, geht wieder Amerika und mit ihm die Welt zurück. Jener Kollege behauptete auch, es wäre manches besser geworden, wenn ich den Ruf nach Berlin nicht abgelehnt hätte. Gewiß am wenigsten durch meine Vorträge; aber ein einziger kann, wenn er mit Kleins Idealismus und Kleins Unverfrorenheit wirkt, bei Berufungsfragen und bei Neuschöpfungen ganz bedeutend ins Gewicht fallen. Mancher, der nicht zu haben war, wäre doch zu haben 
gewesen, wenn man ihn richtig gewollt hätte. Ein kleines Rädchen, das an der richtigen Stelle immer richtig arbeitet, kann viel leisten.

Wenn ich mich bei allen Städten von der Einwohnerzahl Leipzigs so lange aufhalte, werde ich nicht weit kommen; „aber man muß die Einwohner nicht zählen, sondern wägen." Das heißt natürlich ihre geistige Bedeutung.

Nach einigen höchst gemütlichen intimen Diners und einem offiziellen, bei dem ich den sächsischen Unterrichtsminister Seydewitz, unter dem ich zwei Jahre Professor gewesen war, zum erstenmal persönlich kennen lernte, ging es weiter nach Bremen und dann mit einem Hohenzollernschen Fürsten nach New York. Das ist nicht so zu verstehen, als ob ich gewürdigt worden wäre, der Begleiter dieses Fürsten auf einer Amerikafahrt zu sein, sondern derselbe trug mich einfach auf seinen Rücken. Es war Kronprinz Wilhelm bei der Hinfahrt, Kaiser Wilhelm II. bei der Rückfahrt.

Lieber Leser! Meine Eile ist groß, aber die Meerfahrt von Bremen bis New York mit diesem banalen Witz ganz abzutun, bin ich doch nicht imstande. Die großen Ozeandampfer gehören zu dem Bewunderungswürdigsten, was der Mensch geschaffen hat und die Fahrt damit findet man bei jeder Wiederholung wiederum schöner. Das wundervoll brausende Meer jeden Tag wieder anders und jeden Tag wieder staunenswürdiger! Heute weißschäumend, wild stürmend. Siehe das Schiff dort! Nun ist es von den Wellen verschlungen! Nein! Schon taucht der Kiel wieder siegend empor.

Morgen ist das Wetter ruhig, das Meer glatt aber nebelgrau; nebelgrau auch der Himmel, wie man die Melancholie malt. Dann bricht die Sonne aus dem Nebel, und gelbe und rote Funken tanzen auf den Wellen zwischen den tiefschwarzen Flächen der Wolkenschatten, das goldige Licht vermählt sich der Finsternis. Und dann ist wiederum der ganze Himmel blau, und das Meer, azur in weiß, strahlt von so überwältigendem Glanze, daß man die Augen schließen muß. Nur an auserwählten Tagen schmückt es sich mit dem schönsten, dem ultramarinblauem Kleide, eine Farbe so dunkel und doch so leuchtend, mit milchweißem Schaume wie mit Spitzen eingefaßt! Ich lachte einmal, als ich las, daß ein Maler nach einer einzigen Farbe Tage und Nächte suchte; jetzt lache ich nicht mehr darüber. Ich habe beim Anblick dieser Farbe des Meeres geweint; wie kann uns eine bloße Farbe weinen machen? Dann wieder Mondesglanz oder Meerleuchten in pechschwarzer Nacht! Um von allen diesen Herrlichkeiten einen Begriff geben zu können, müßte man Maler sein und dann könnte man es erst recht nicht.

Wenn eines unserer Bewunderung noch mehr wert sein kann als diese Naturschönheit, so ist es die Kunst des Menschen, welcher in dem seit den Zeiten der Phönizier und noch viel länger geführten Kampf mit diesem unendlichem Meere so vollständig siegte. Wie unbarmherzig der Kiel die Flut durch- 
schneidet, wie der Meergott wild aufschäumt unter der bohrenden Schraube! Fürwahr, das höchste Wunder der Natur, das ist der kunstfertige Geist des Menschen!

Wenn ich wie einst Solon um den Glücklichsten der Sterblichen gefragt würde, ich würde ohne Zagen Kolumbus nennen. Nicht als ob nicht andere Entdeckungen der seinen gleichkämen, schon die des Deutschen, Gutenberg. Aber das Glück ist mitbedingt durch die sinnliche Wirkung und die muß bei Kolumbus am höchsten gewesen sein! Ich kann nie in Amerika landen, ohne ein gewisses Gefühl des Neides gegen ihn oder besser vielleicht der Beseligung, daß ich einen kleinen Teil seiner Freude mitempfinden kann. Freilich fuhr Kolumbus nicht mit dem Kronprinz Wilhelm, er sah auch New York nicht mit dem leiblichen Auge, aber mit dem geistigen hat er vielleicht mehr gesehen als wir, das New York nach 100, 200 Jahren!

Dafür ist auch Kolumbus das Prototyp der Entdecker geworden. Sein „immer, immer nach West" für ihre Ausdauer, sein „Land, Land" für die Freude des Gelingens, und seine ganze Tat für die Überzeugung, daß das Leben der Güter Höchstes nicht ist. „Setzest du nicht das Leben ein, nie kann dir das Höchste gewonnen sein."

Nicht nur der Schönheitssinn, auch alle anderen finden bei der Meerfahrt volle Befriedigung. Eine reichliche und gute Küche sorgt für den Geschmack, ein ganz nettes Orchester für das Gehör. Da marschieren wieder oft unsere Wiener Komponisten auf, freilich nicht die ganz großen, aber Strauß, Ivanovici, Waldteufel u. v. a. Lebhaft beklatscht wurden die „Donauwellen“ an den Wellen des atlantischen Ozeans und in der Tat, denken wir an Haydn, Mozart, Schubert, Beethoven, so können wir, wie Schiller von der Ilm auch von der Donau sagen, daß ,ihre leiseren Wellen im Vorüberziehen manch unsterbliches Lied erlauscht haben."

So gibt es nichts Behaglicheres, als das Schiffsleben, besonders für den, dem ein Gott gegeben, von der Seekrankheit verschont zu bleiben und ruhigen Gemütes auf so viele Dahingestreckte blicken zu können. Der Spaß erreicht seinen Höhepunkt, wenn vermöge irgend einer hydrodynamischen Zufälligkeit plötzlich unerwartet eine Welle über Bord spritzt und die im Halbschlummer Dahingestreckten unter Gekreisch aufspringen.

Wenn ich dann im Hafen von New York einfahre, so erfaßt mich immer eine Art Rausch. Diese turmhohen Häuser und die alles überragende Statue der Freiheit mit der Fackel! Dabei dieses Pfeifen und Singen der Schiffe durcheinander; das eine schroff warnend, das andere erschreckt aufschreiend, das eine munter pfeifend, das andere in Quarten melancholisch jammernd; dort erschallen gar die unnachahmlichen Töne der Sirenen! Wenn ich ein Musiker wäre, ich würde eine Symphonie komponieren: Der Hafen von New York.

Doch damals hatte ich keine Zeit zur Sentimentalität. Ich hatte sofort 
in Hoboken einen Cab engagiert, der mich zuerst in die Office der Southern Pacific Railroad, dann von dort direkt auf den Bahnhof bringen sollte; alles für 3 Dollar. Aber in der Office erfuhr ich, daß der besonders beschleunigte Zug, für den ich ermäßigten Preis hatte, nur zweimal in der Woche fährt und ich zwei Tage in New York warten müsse. So beschied ich denn meinen Wagen ins Westminster-Hotel und hatte Zeit, mich zwei Tage in New York herumzutreiben.

Langweilig ist es dort auch nicht. Welch reichen Stoff der Unterhaltung und Beobachtung bietet nur eine einfache Tramwayfahrt! Fahrkarten werden nicht ausgegeben, Überfüllungsverbote und verschiedene Preise gibt es nicht. Der Kondukteur entdeckt mit Falkenblick jeden neu Einsteigenden; dieser drückt ihm 5 Ct. in die Hand, ein Zug an einer Schnur und die Zahlung ist an einem Zählwerk, das sich oben im Wagen befindet, quittiert. Gleichzeitig ertönt ein Glockenschlag, den alle Anwesenden hören. Wenn man einen Platz in der Nähe des Kutschers hat, so kann man Führergaben bewundern, die unmöglich denen eines Napoleon I. oder Moltke viel nachstehen können. Wie rasend schnell eine freie Strecke durchmessen wird, wie vor einem Automobil sofort gehalten wird (denn diese fahren einfach in scharfem Bogen an den Kutschen und Tramwaycars vorüber), alles das möge man sich in Natur ansehen und noch einiges andere, was in New York sehenswert ist.

Am dritten Tage gings dann um so schneller vorwärts. In vier Tagen und vier Nächten kam ich von New York nach San Franzisko. Man wird einfach fortgeschleudert, gewissermaßen fortgeschossen. Die Püffe, die man beim Gehen durch den endlos langen Zug nach dem Speisewagen, Aussichtswagen usw. erhält, sind nicht gerade angenehm. Die Aussichtswagen sind rückwärts ganz offen, man kann sich auf das Abschlußgitter setzen oder darüber hinausbeugen und hat dann einfach acht zu geben, daß man nicht bei einem jähen Stoße hinunterfällt.

Die Landschaft war freilich meist einförmig, doch ist schon die direkte Beobachtung der Schnelligkeit der Fahrt interessant. Wenn man vom Aussichtswagen aus nach rückwärts blickt, so erscheinen die Eisenbahnschienen wie ein endloses Band, das mit rasender Geschwindigkeit unter dem Wagen hervorgezogen wird. Interessant war auch die Fahrt auf dem riesigen Holzdamm mitten durch den Salzsee und die ausgedehnten von Salzkristallen wie mit Schnee bedeckten Felder vor und nach demselben. Gegen das Ende der Fahrt ist der Übergang über die Sierra-Nevada wunderschön. Er erinnert an den Semmering, freilich nicht ganz so malerisch, aber noch viel großartiger an Länge der Strecke und Höhe der Berge.

Dank meiner Verzögerung in New York kam ich nach Berkeley zu spät. Die Sommerschule begann am 26. und ich traf erst am 26. abends ein. Da freilich dieser Tag bloß mit den einleitenden Reden, Inskriptionen usw. vertändelt 
worden war, so hätte ich keine einzige Stunde versäumt, wenn ich tags darauf um 9 Uhr morgens begonnen hätte. Allein dazu erklärte ich mich außerstande. Die Wirkung des viertägigen Schütteins und Schleuderns trat nämlich erst jetzt zutage. Ich konnte auf ruhigem Boden keinen Schritt mit Sicherheit gehen und nachts im Bette erwachte ich fortwährend vor Schrecken, daß ich nicht geschüttelt wurde und doch vom Schütteln träumte.

Nun muß ich gestehen, daß ich ein wenig Lampenfieber immer vor der ersten Vorlesung habe; hier gar, wo ich englisch sprechen sollte. Ich hatte auf der Reise weniger Gelegenheit zur englischen Konversation gehabt, als ich gehofft hatte. Die Deutschen, die englisch gekonnt hätten, sprachen nach einigen englischen Worten wieder deutsch und die echten Engländer sprachen überhaupt gar nichts.

Meine englische Konversation ging nach diesem Schema. Ich: When lunch will be served? Er: ieeöö. Ich: I beg you, could you say me, at what hour lunch will be served? Sein Gegurgel ist jetzt um eine gute Quint tiefer: aoouu. Ich begreife das Verfehlte meines Angriffsplanes und schreie verzweifelt: Lönch, lanch, lonch, launch usf. Ich bringe Vokale hervor, die man in Gutenbergs Setzkasten vergebens suchen würde. Jetzt zeigt sein Gesicht einiges Verständnis; Ah, loanch? Nun ist die Brücke der Verständigung geschlagen. Ich: When? at what hour? When o'clock? Er: Half past one! Wir haben uns verstanden. Und nun sollte ich in dieser Sprache dreißig Vorlesungen halten! Ich erklärte mich also Dienstag den 27. Juni außerstande und begann erst Mittwoch. Bei der ersten Vorlesung war ich wohl zaghaft, aber bei der zweiten schon unbefangener und als ich vollends hörte, daß mich die Studenten gut verstehen, ja meine Darstellung sehr übersichtlich und klar finden, da fühlte ich mich bald ganz zuhause.

Ich kann nicht umhin, meiner englischen Sprachlehrerin in Wien Miß May O'Callaghan für diesen Erfolg meinen Dank auszusprechen. Ohne ihre unermüdlichen Bemühungen meiner widerstrebenden Zunge nachzuhelfen, hätte ich ihn nicht erzielt. Mit welchem Stolze wandte ich wie selbstverständlich die Worte Blackboard und Chalk an, als ich mir schreibfähige Kreide und eine ausreichende Schreibtafel verschaffen mußte! Wie gut klappte die Aussprache von Algebra, Differenzialcalculus, Chemistry, natural Philosophy usw.!

Ja auch einen ausgezeichneten Hummersalat verdanke ich meinem Fleiße. Da stand auf der Speisekarte lobster-salad. Ich erinnerte mich sogleich der Lektion, wo ich kaum glauben konnte, daß Hummer lobster heißt; also her mit dem lobster und er mundete ganz vorzüglich.

Die Universität Berkeley, wo ich zu wirken hatte, ist das Schönste, was man sich denken kann. Ein Park von einem Quadratkilometer Fläche, mit 
Bäumen, die gewiß schon Jahrhunderte sahen, oder sind es Jahrtausende? wer wüßte das so geschwind! Darin schöne, modern eingerichtete Gebäude, freilich schon vielfach zu klein; aber man macht Neubauten, Bauplatz und Geld ist ja da.

Es liegt ein gewisser philosophischer Hauch darüber. Der Name Berkeley ist ja der eines hoch angesehenen englischen Philosophen, dem man sogar nachrühmt, der Erfinder der größten Narrheit zu sein, die je ein Menschenhirn ausgebrütet hat, des philosophischen Idealismus, der die Existenz der materiellen Welt leugnet, also Idealismus in einem anderen Sinne, als ich das Wort gebrauchte. Die Philosophie hat dort ein eigenes Lehrgebäude; nicht ein Lehrgebäude aus Phrasen und Hirngespinsten, Pardon, ich wollte sagen aus logischen Schlüssen und Vernunftbegriffen, sondern ein veritables Gebäude aus Stein und Holz, wo mit Stimmgabeln, Farbenscheiben, Kymographien und Registriertrommeln die Psyche erforscht wird.

Wichtiger war für mich ein anderes Gebäude. Ein spekulativer Wirt hatte nämlich im Konversationslexikon gelesen, daß Berkeley ein englischer Bischof war, dessen Residenz Cloyne Court hieß, und daraufhin ein Professorenwirtshaus gebaut, das er Cloyne Court nannte und wo auch ich wohnte. Auf eine gewisse äußere Ähnlichkeit mit einem alten englischen Bischofsitz hatte er keinen Wert gelegt; es lag ja in der Euklid-Avenue und hatte exakt Parallelepipedform ohne jede Spur von etwas nichteuklidischem. Aber im Innern war es komfortabel. Ich hatte ein kleines Schlafzimmer, ein etwas größeres Arbeitszimmer und ein Badezimmer, alles elektrisch beleuchtet. In den Zimmern konnte man durch einige dicke Röhren warmes Wasser zirkulieren lassen und so eine mäßige Heizung bewirken, was im Juli unter der Breite von Palermo oft willkommen war, so eisig ist manchmal der vom pazifischen Ozean wehende Wind. Dagegen ist in Berkeley der Winter nur wenig kälter als der Sommer; nur reich an Regen, der im Sommer gänzlich fehlt.

Das Essen war gut. Wenigstens von einer der vorgesetzten Speisen konnte man in der Regel etwas hinunterwürgen. Gedruckte Speisekarten gab es nicht. Das Menü wurde von den meist Augengläser tragenden Kellnerinnen vor jeder Mahlzeit herabgeleiert, so daß es mehr wie ein monotones mit gedämpfter Stimme gesungenes Lied klang.

„Doch des Lebens ungemischte Freude ward keinem Irdischen zuteil“, auch nicht auf einer Reise nach Berkeley. Zuerst soll der Magen das Wort behalten. Ich hatte bisher weder aus offenen noch aus den mit Flußwasser und Kohlensäure gefüllten verschlossenen Flaschen Wasser getrunken, und so meinen Magen trotz der — ungewohnten Nahrung gesund erhalten. Aber Berkeley ist abstinent; Bier und Wein zu trinken oder auszuschenken ist strengstens verboten. Verdursten wollte ich doch nicht; ich versuchte also das Wasser, aber ohne Eis, vielleicht ist es in Berkeley gesünder als in New 
York und St. Louis. Leider nicht! Mein Magen revoltierte, und als ich einmal die ganze Nacht die Kleider anbehalten mußte, um sie nicht zu oft aus- und wieder anziehen zu müssen, wagte ich an einen Kollegen die Frage nach einer Weinhandlung. Die Wirkung erinnerte mich an eine Szene im Rauchkoupé der Eisenbahn zwischen Sacramento und Oakland. Es hatte sich zu uns ein Indier gesellt, welcher ganz naiv nach einem — weil es ein Indier war, sagen wir Bajaderenhaus in San Franzisko fragte. Die meisten im Rauchkoupé waren aus San Franzisko und Mädchen mit der Devise: ,give me money, I give you honey" gibt es in San Franzisko gewiß; aber alle machten verdutzte, verlegene Gesichter. Ein ganz gleiches Gesicht machte mein Kollege auf die Frage nach einer Weinhandlung. Er sah sich ängstlich um, ob niemand zuhöre, maß mich, ob er mir ganz trauen dürfe und rückte endlich mit einer ausgezeichneten Handlung kalifornischen Weines in Oakland heraus. Es gelang mir richtig, eine ganze Batterie Weinflaschen einzuschmuggeln und der Weg nach Oakland wurde mir seit dieser Zeit sehr geläufig. Auch mein Magen sagte Amen dazu und erholte sich bewunderungswürdig schnell, obwohl meine Kost sonst dieselbe blieb. Aber mein Glas Wein nach Tisch mußte ich sorgfältig versteckt trinken, so daß ich fast selbst das Gefühl bekam, einem Laster zu fröhnen. Die Temperenz ist also auf dem besten Wege, die Heuchelei, deren es doch auf der Welt schon genug gibt, um eine neue Spezies zu vermehren.

Kaum war mein Magen beschwichtigt, so stellten sich andere Übel ein. Mein lästiges Asthma verschwand bisher immer, sobald ich den Fuß auf den Dampfer setzte und blieb verschwunden, bis ich wieder europäischen Boden betrat. So auch diesmal, bis ich nach Kalifornien kam; aber, was man an dessen Klima rühmt, die feuchte Kühle, das lockte mir den ungebetenen Gast, das Asthma, wieder auf den Hals,

Dann bekam ich unter dem Arme eine Beule (ich glaube infolge eines neu gekauften Hemdes, das ich ungewaschen angezogen hatte). Ich mußte sie mir im Roosevelt-Hospital aufschneiden lassen. Es war von höchstem Interesse, das amerikanische Hospital so gründlich kennen zu lernen, das an Eleganz dem Kaiser Wilhelm II. (ich meine natürlich das Dampfschiff) nicht nachsteht, aber kostete 35 Dollar. Es war dieses der kostspieligste Luxus, den ich mir auf der ganzen Reise, erlaubte, und raubte mir ein weniger zweifelhaftes Vergnügen.

Dienstag den 4. Juli war nämlich independence-day, der größte amerikanische Feiertag, und da ich Samstag und Sonntag keine Vorlesung hatte, so brauchte ich nur die am Montag auszulassen oder später nachzuholen, um vier Tage für einen eiligen Besuch des Yosemite-Tales zu erübrigen. Das mußte ausfallen; dafür hörte ich Sonntag den 2. Juli die half hour of music, die wie jeden Sonntag im griechischen Theater gratis zum besten gegeben 
wurde. Dieses Theater ist eine getreue Kopie des Sophokleischen Theaters in Athen, nur, wie mir schien, noch vergrößert. Da es in Berkeley im Sommer nie regnet und doch auch wegen des häufigen Nebels die Sonne wenig scheint, so tut das vollständig unbedeckte Theater gute Dienste. Nur die Musik war in dem architektonisch wunderschönen, rings von Eukalyptus und lifeoaks umrahmten Raume unendlich dünn. Da hätte Mahler mit den Philharmonikern hingehört, die dritte Symphonie spielend, so daß die Bäume vor Wonne gezittert hätten und der stille Ozean aufhorchend noch stiller geworden wäre; die Menschen dort hätten es ja doch nicht verstanden.

Am Dienstag sah ich vom Dache des Cloyne-Court aus die großartigen Feuerwerke an, die, wie alljährlich, zur Feier des independence-day abgebrannt wurden. Cloyne-Court liegt nämlich auf der Anhöhe und gewährt eine Aussicht auf die Bai von San Franzisko, das goldene Tor, den MountTamalpais usw., wie sie der alte englische Bischof von seinem Cloyne-Court aus kaum schöner hatte.

Der liebe Gott selbst schien an der Feier sein Wohlgefallen zu haben; denn er ging bei Sonnenuntergang mit einem Feuerwerke voran, seiner eigenen Größe und der seiner Schöpfung würdig. Da hatte ich wieder, wie schon oft auf der Amerikareise, den Wunsch, malen zu können!

Als die letzten Strahlen des Abendrotes verschwunden waren und die Lichter von San Franzisko hell über die Bai herübergrüßten, begannen die Feuerwerke der Menschen. Bald flammte wunderbar zu unseren Füßen ein buntes Licht auf, bald erstrahlte weit am Horizont ein glühender Stern. Wo soll man hinsehen? In Berkeley und San Franzisko leuchtet und flammt es; aber dort in Oakland, welch schöner Effekt! Kaum sieht man hin, hat man einen noch schöneren in Alameda versäumt. Ich nahm mir vor, jeden Abend des 4. Juli in meinem Garten ein kleines Feuerwerk abzubrennen. Der Kampf Washingtons und seiner Schar hat doch nicht bloß lokalpatriotische, sondern vielmehr welthistorische Bedeutung.

Schiller sagt einmal: „Noch ein paar tausend solche Kerle wie ich, und aus Deutschland soll eine Republik werden, gegen die Rom und Sparta Nonnenklöster waren." Das blieb freilich aus. Ein paar tausend Kerle wie du? Die Welt hat nicht einen mehr gezeugt. Aber Ideen sterben nicht. Die Republik, im Vergleich mit der Rom und Sparta Nonnenklöster waren, existiert, freilich jenseits des Ozeans, und wie kolossal ist sie und wächst sie! „Die Freiheit brütet Kolosse aus."

In der Folgezeit war ich jeden Samstag und Sonntag irgendwo eingeladen. Das erste Mal bei Mrs. Hearst auf ihrem prachtvollen Landsitze in der Nähe von Livermore. Wer ist Mrs. Hearst? Es ist nicht ganz leicht, das einem Europäer klar zu machen. Am nächsten würde man der Wahrheit kommen, 
wenn man sagte, sie ist die Universität Berkeley. In Europa ist die alma mater eine antike Idealgestalt, in Amerika ist es eine wirkliche Dame und, was am wichtigsten ist, mit wirklichen Millionen, von denen sie alljährlich so etliche zur Erweiterung der Universität hergibt; auch meine Amerikareise wurde natürlich aus ihrem Gelde bezahlt. Der Präsident der Universität (was wir den Rektor nennen würden, er ist es aber lebenslänglich) ist nur das ausführende Organ der Trustees, an deren Spitze Mrs. Hearst steht. Der gegenwärtige $^{1}$ mußte sich bei seiner Ernennung eine Reihe von Freiheiten ausbedingen, um doch einiges aus eigener Machtvollkommenheit für die Universität tun zu können.

Noch schlimmer — doch wie kann ich sagen schlimmer? wer, wie ich der Gastfreundschaft Mrs. Hearsts so schöne Stunden verdankt, wie sollte der behaupten, daß eine solche alma mater etwas schlimmes wäre? Also noch schroffer sind die Verhältnisse an der Leland Stanford junior University in Palo Alto, welcher ich einen eintägigen Besuch abstattete.

Mr. Leland Stanford senior hatte den Bau der ersten Pazificbahn geleitet, das heißt der ersten Bahn, welche die kontinuierliche Bahnverbindung zwischen dem atlantischen und stillen Ozean herstellte. Er wußte im Kongresse, wo er auch einflußreich war, die Wichtigkeit des Unternehmens ins rechte Licht zu setzen und zu veranlassen, daß dieser beschloß, die Hälfte der Baukosten zu tragen, wofür er sich gewisse Hoheitsrechte vorbehielt, die Einnahmen aber im Wesen dem Bahnunternehmer überließ. Herr Stanford gründete nun eine Gesellschaft von ganz anderem Namen, deren Haupt er wieder war, welche der Eisenbahnbaugesellschaft alle Materialien und Arbeitskräfte zu liefern hatte. Da er das Haupt der einen und anderen Gesellschaft war, so erreichte er leicht, daß die erste von der zweiten alles um den doppelten Preis kaufte und der Staat nominell die Hälfte, faktisch das ganze bezahlen mußte, er aber die ganzen Einnahmen hatte.

Als er enorm reich geworden war, raffte ein plötzliches Unglück seinen einzigen Sohn, für den er alles gesammelt hatte, hinweg. Er und besonders Mrs. Stanford verfielen in eine Art religiösen Wahnsinn. In Europa, wenn eine alte Dame etwas überschnappt, kauft sie sich ein Dutzend Katzen oder einen Papagei; hier ließ sie Baumeister ersten Ranges berufen (was bekommt man nicht um Geld) und erbaute eine Universität, die sicher noch der Segen künftiger Geschlechter sein wird.

Während die Universität Berkeley im Pavillonsystem erbaut ist, so ist die Stanford-Universität nach einem einheitlichen, architektonisch schön wirkenden Plane angelegt, der mir freilich für den Unterricht unzweckmäßig vorkommt. Die Architekten sind eben überall gleich. Besonders prachtvoll ist

\footnotetext{
${ }^{1}$ Benjamin Ide Wheeler
} 
die Universitätskirche mit Wand- und Deckengemälden, Glasmalereien und Kunstwerken der Plastik reich geschmückt. Die Orgel, die in meiner Gegenwart gespielt wurde, klang so wundervoll, daß ich bei diesen Tönen gerne andächtig war.

Nach dem Tode ihres Gemahls war Mrs. Stanford lange allein die Universität. Nun starb auch sie, nicht ohne vorher in ihrem Testamente für die Universität ausgiebig gesorgt zu haben.

An der Stanford-Universität soll einmal ein Professor der Nationalökonomie in seinen Vorlesungen gegen den Gründerschwindel losgezogen haben; der Präsident glaubte sich bei Mrs. Stanford ein Blatt einzulegen, wenn er diesen Professor sofort entließ; aber Mrs. Stanford selbst war so hochherzig, ihn zurückzuberufen und den übereifrigen Präsidenten zu tadeln.

Daß an solchen Universitäten das männliche und weibliche Element unter den Studenten und im Lehrkörper gleichberechtigt ist, versteht sich von selbst, und ich will nur einen drastischen Beleg für die weitgehende Herrschaft des Weiblichen anführen. Eine meiner Kolleginnen im Lehramte, ich behielt ihren Namen, Miß Lilian Seraphine Hyde, eine nicht unebene Dame, las ein ganz in gleicher Weise wie das meine angekündigtes, wie wir sagen würden, zweistündiges Kolleg über die Zubereitung von Salaten und Dessert. Ich kann den Lektionskatalog noch heute vorzeigen.

Alle Räume der Universität wimmeln von Damen, die an Zahl den männlichen Studenten nicht viel nachstehen dürften. Besonders fällt es auf, daß in jedem Raume ein Damenhut deponiert zu sein pflegt. Im Professorenzimmer ein Damenhut, in dem Raum, der als Waschzimmer, Telephonkammer und noch etwas dient, ein Damenhut, in der Dunkelkammer ein Damenhut; ja als ich nach der schon erwähnten Operation etwas geschwächt und konfus fortging, hätte ich in der Zerstreutheit bald statt meines Hutes einen dort deponierten Damenhut aufgesetzt.

Doch nun zurück zu Mrs. Hearst, der alma mater berkeleyensis. Sie hatte mich, wie schon erwähnt, mit einer Reihe anderer an der Sommerschule lehrenden Professoren nach ihrem Landsitze in der Nähe von Livermore geladen, einem Juwel, wie es Luxus, Reichtum und guter Geschmack nur in so verschwenderisch ausgestatteter Natur zu schaffen vermögen. Auf der Bahnstation empfingen uns die Kutschen und bald ging es durch ein stark phantastisches aber nicht unschönes Eingangstor nach einem Parke von fabelhafter Baumpracht und Blumenschönheit. Der Reichtum setzt sich hier in Wasser um, und wo dieses nicht gespart wird, ersteht in Kalifornien ein Blumenflor, der Sommer und Winter gleichmäßig fortblüht. Lange, doch für mich noch zu kurz, durchquerten wir den Park, der auch die schönsten Aussichtspunkte nach dem Mount Diable und Mount Hamilton bietet. Endlich erreichten wir das Wohnhaus. Es ist in portugiesisch-mexikanischem Stile erbaut, ein 
Kranz von Gebäuden rund um einen durch schwere eiserne Tore verschlossenen Hof; offenbar eine Art Festung. Den Mittelpunkt des Hofes bildet ein antiker Marmorbrunnen, den die Besitzerin selbst in Verona kaufte und bis ans stille Meer hatte transferieren lassen. Nach ihm heißt der ganze Landsitz "Hazienda del pozzo di Verona“.

Mein Nachbar im Wagen erklärte mir, daß die Besitzerin eigens einen deutschen Architekten namens Schweinfurt berufen hatte, der nach dem Studium aller alten spanischen und portugiesischen Gebäude in Mexiko dieses geschaffen hat. Ich bemerkte: „Der muß einen guten Geschmack gehabt haben!" worauf mein Begleiter erwiderte: „Ja, er ist auch daran gestorben." Ich frug: „Wie ging denn das zu?" Darauf er: „Die kalifornischen Weine schmeckten ihm zu gut und da trank er fort, bis er starb." Diese Kalifornier haben eine entsetzliche Idee von ihren, allerdings sehr starken Weinen. Am Ende war es nicht einmal so arg. Ich werde ja auch einmal sterben und dann zu trinken aufhören, so daß ich also auch fortsaufen werde, bis ich sterbe.

Das Innere der Hazienda ist ein Schatzkästlein voll der herrlichsten Kunstwerke und Seltenheiten, die die Besitzerin in allen Gegenden der alten und neuen Welt zusammengekauft hat, die originellste Mischung griechischer und römischer, mittelalterlicher, mexikanischer, chinesischer, japanischer und indischer Seltenheiten.

Bei Tisch saß ich, als einziger anwesender Europäer zur Rechten der Mrs. Hearst. Das erste Gericht waren Brombeeren. Ich dankte. Dann kam eine Melone, welche die Hausfrau eigenhändig für mich recht appetitlich gesalzen hatte. Ich dankte wieder. Dann kam oat-meal, ein unbeschreiblicher Kleister aus Hafermehl, mit dem man in Wien vielleicht die Gänse mästen könnte; ich glaube aber eher nicht, denn Wiener Gänse würden das kaum fressen. Ich hatte aber schon beim Abweisen der Melone einen etwas ungnädigen Blick der alma mater bemerkt. Auf ihre Küche ist ja auch eine alma mater stolz. Ich würgte also mit abgewandtem Gesicht und danke Gott, daß mir nichts Menschliches dabei passierte. Das ist das Unangenehme bei Einladungen in Amerika; in Gasthäusern darf man, was man nicht essen kann, stehen lassen; aber was macht man gegenüber einer Hausfrau, die auf die Güte der amerikanischen Küche im allgemeinen und der ihren im besonderen stolz ist? Glücklicherweise kam dann noch Geflügel, Kompott und manches, womit ich den Geschmack wieder decken konnte.

Nach Tisch ging man in das Musikzimmer, ein Raum, wenn ich recht schätze, ungefähr so groß, wie der Bösendorfer Saal, aber welche phantastischbarocke Ausschmückung! An Schönheit wüßte ich ihm keinen der kleineren Wiener Konzertsäle zu vergleichen. Die Kunde von meinem armseligen Klavierspiel war bis in die Hazienda gedrungen. Ich wurde aufgefordert, das Konzert zu eröffnen. Nach einigem Sträuben setzte ich mich an den Flügel, 
einen Steinway von der allerhöchsten Preislage. Ahnungslos griff ich in die Tasten; einen Flügel von solcher Klangschönheit hatte mein Ohr vielleicht schon in einem Konzerte gehört, nie mein Finger berührt. Wenn mich je die Strapazen meiner kalifornischen Reise gereut hätten, von jetzt an nicht mehr. Ich spielte eine Sonate von Schubert, anfangs freilich war mir die Mechanik etwas fremd, aber wie leicht gewöhnt man sich an das Gute! Schon der zweite Teil des ersten Satzes ging gut und im zweiten Satze, einem Andante, vergaß ich mich selbst; nicht ich spielte die Melodie, sondern diese lenkte meine Finger. Ich mußte mich mit Gewalt zurückhalten, nicht auch noch das Allegro zu spielen und das war gut, denn dort wäre meine Technik abgefallen. Nach mir spielte eine Schülerin Barths in Berlin mit ebensoviel Technik als Musikverständnis. Unter den Anwesenden war auch ein Professor der Musik in Milwaukee ${ }^{2}$, eine martialisch männliche Gestalt, sicher ein vortrefflicher Bärenjäger; aber auch musikalisch gründlich gebildet. Er hatte ebenfalls bei Barth Klavierspiel betrieben, man kann nicht sagen, gelernt. Er wußte, daß Beethoven neun Symphonien geschrieben hat und daß die neunte davon die letzte ist. Mir tat er unverdiente Ehre an; denn gelegentlich einer Debatte, ob Musik auch humoristisch sein könne, ersuchte er mich, das Scherzo aus der neunten vorzuspielen. Sollte ich sagen, ich kann es nicht, einem Professor in Milwaukee gegenüber? Da ward auch ich humoristisch und sagte: „Gerne, nur bäte ich ihn, die Pauke zu spielen, es nimmt sich besser aus, wenn die ein zweiter hineinspielt." Darauf wurde er mit seiner Bitte still.

Die Nacht schlief ich in der Hazienda in einem wundervollen Schlafzimmer, verbunden mit Badezimmer, mit einem Mohren zu meiner ausschließlichen Bedienung, der auch die Stiefel putzte. Gerade über meinem Bette hing das Bild eines Schutzengels von idealer Schönheit. Ich liebe die Kunst besonders, wenn sie zugleich einer Idee dient. Was soll mir in Wohnräumen ein noch so schönes Bild der Schlacht von Abukir? Aber ein Schutzengel zu Häupten der Ruhestätte spricht gewissermaßen den Wunsch des Hauswirts aus, daß ich bei ihm gut schlafen möge. Ja, ich bin abergläubisch. Gerade damals litt ich stark an Asthma und hatte schon Bedenken, nach der Hazienda zu fahren. Dieser Schutzengel tröstete mich und wirklich war die Tücke des Asthma von dieser Nacht an gebrochen.

Am nächsten Tage hatte es erst mit dem Besehen von Merkwürdigkeiten in Haus und Hof, Wald und Feld kein Ende. Wir fuhren unter anderem zu riesigen life oaks mit ungeheuren weitausgebreiteten Ästen. Über den Zweigen einer derselben war eine ganze Hütte gebaut, zu der eine Stiege hinaufführte, eine Art Hundinghütte im ersten Stockwerke. Erst nachmittags fuhr ich zurück und gelangte spät abends nach Berkeley, um am nächsten Tage wieder

\footnotetext{
${ }^{2}$ Albert A. Stanley
} 
rechtzeitig im Hörsaale erscheinen zu können.

Der nächste Samstag und Sonntag war der Licksternwarte gewidmet. Ich fuhr schon Freitag Nachmittag nach dem freundlichen Städtchen San-José, in dem manche Straßen Palmenalleen sind. Dort wandelt man nicht nur unter Palmen, man fährt unter Palmen Tramway, Bicykle und Automobil. Nächsten Tag um sieben Uhr morgens trat ich in der etwas defekten Postkutsche die Fahrt auf den Mount-Hamilton an, der etwa die Meereshöhe des Semmering hat, aber höher erscheint, da der Ausgangspunkt der Fahrt nur wenig oberhalb des Meeresniveaus liegen dürfte. Die Straße ist sehr gut und führt in Serpentinen durchaus langsam und gleichmäßig ansteigend zwischen Weinund Obstgärten, auch Wald und Wiesen hinan. Auf letzteren steht aber um diese Jahreszeit nur Heu. Dort fressen die Kühe im Sommer Heu und im Winter frisches Gras.

Mein Kutscher, ein knorriger, brummiger Alter, ist zugleich Postbeamter. Gleich nach Verlassen des Gasthofes werden mit viel Gebrumm die Postsendungen sortiert, die in einigen Säcken vor unseren Füßen liegen. Bald sind wir außerhalb der Stadt. Draußen vor dem Tor eines großen umzäunten Anwesens begrüßt uns ein hübscher Hund mit munterem Bellen. Mein Kutscher steckt ein paar Briefe in ein Zeitungspaket, und wirft dieses geschickt dem Köter ins Maul, der damit sofort unter dem Zaune hinwegschlüpft. Diese Art Postdienst wiederholt sich bei sehr vielen Anwesen. Bei anderen ist eine Holzstange mit einem großen Nagel aufgepflanzt. Mein Kutscher nimmt geschickt, ohne den Wagen anzuhalten, die für die Absendung bestimmten Postsachen herab und hängt die angekommenen darauf. Nur zweimal, wo Körbe mit Eßwaren oder andere große Pakete aufzugeben waren, warteten Mägde, deren Menschenrasse anzugeben ich zu wenig Anthropologe bin. Wir hatten zweimal Pferde gewechselt und einmal geluncht (aber fragt mich nur nicht wie?).

Etwa um 1/2 2 Uhr kamen wir oben bei der Sternwarte an. Nur die jüngeren Astronomen, an der Spitze Dr. Tucker, waren anwesend, da Direktor Campbell mit den älteren schon in Spanien die Beobachtung der totalen Sonnenfinsternis vorbereitete. Da ich damals selbst noch willens war, mir diese anzusehen, fragte ich Dr. Tucker, wo die Finsternis beobachtet werde. „In Daroca-Ateca-Almazan“, antwortete er. Ich erschrak ein wenig und sagte unwillkürlich, das ist für mich ein spanisches Dorf. Er aber entgegnete ruhig, es ist in der Tat ein spanisches Dorf nordöstlich von Madrid. Ich weiß nicht, der Name machte keinen günstigen Eindruck auf mich, Spanien fing doch an, mir etwas spanisch vorzukommen.

Drauf zeigte man mir alle die Hilfsmittel der herrlich eingerichteten Sternwarte, welche wegen der günstigen Lage derselben auch auf das Fruchtbarste ausgenützt werden können. Das großartigste ist das Riesenteleskop mit der 
von Alvan Clark geschliffenen 28 zölligen Linse, (the big glass sagen sie einfach), mit dem eine der interessantesten astronomischen Entdeckungen der Neuzeit, die der beiden Marsmonde, gemacht wurde. Im Innern des riesigen Pfeilers, der das Teleskop trägt, ist der Bürger Lick begraben, der die ganze Sternwarte aus seinen Privatmitteln erbauen ließ. Ist das nicht idealistisch? Ich durchschaue ihn. Er wußte gewiß, daß es für ihn gleichgültig ist, wo seine Gebeine ruhen; aber der Welt wollte er ein sinnfälliges Zeichen geben, was das letzte Ziel eines Millionärs sein soll. Fürwahr! Er hat sich für sein Geld die Unsterblichkeit gekauft.

Wenn ich ein Dichter wäre, ich möchte unter dem Titel „Zwei Idealisten“ das Zusammentreffen Schillers und Licks im Himmel schildern. Schiller läßt die Weisheit zum Reichtume sagen: Ich brauch' dich nicht. Lick beweist das Gegenteil. Freilich die Begeisterung, die man um Geld bekommt, ist nur eine Begeisterung zweiter Güte; die Liebe, die man um Geld bekommt, nicht einmal dritter Güte; aber einen Steinwayflügel bekommt man um Geld, eine Amatigeige, einen Böcklin und nun auch die Unsterblichkeit.

Ich will hier noch eine Geschichte erzählen, die an Idealismus und Gelderwerb anknüpft. Der große amerikanische Physiker Rowland hatte eine Rede gehalten, daß der Gelehrte nicht nach Gelderwerb streben dürfe. Ein Jahr darauf wird er unwohl, lässt sich ärztlich untersuchen und erfährt, daß er höchstens mehr drei Jahre zu leben habe. Er hatte eine Frau und vier unversorgte Kinder. Im Widerstreite der Pflichten siegt die Familienliebe. Er erfindet einen Typendrucktelegraphen, läßt ihn patentieren und erringt so, als er wirklich bald darauf starb, seiner Witwe ein Vermögen von 200000 Dollar, also etwa eine Million Kronen; aber das Prinzip seiner Rede straft er selbst Lügen. Weißt du, lieber Leser, was ich an Rowland am meisten bewundere? Daß er gleich eine so einträgliche Entdeckung bei der Hand hatte. Möge er im Himmel auch Schillern die Hand reichen!

Der amerikanische Kaufmann freilich ist beim Gelderwerbe streng realistisch. So konnte es ein sehr verständiger Kaufmann, dem ich meinen Reisezweck auseinandersetzte, absolut nicht begreifen, warum ich bis San Franzisko reise, wenn meine Einnahmen nur die Reisekosten decken.

Ich hatte vor dem Pfeiler, der auf seinem Scheitel das Riesenfernrohr, in seinem Bauche die Gebeine des Bürgers Lick trägt, geträumt. Nun ging's weiter durch alle Räume der Sternwarte. Ein reiches Material wird hier bestens ausgenützt. Jede Zone des Himmels hat ihren eigenen Kasten, in diesem jeder Stern seine eigene Schublade, damit alle Beobachtungen gleich wiedergefunden und benutzt werden können; der Inhalt der Laden wächst rapid an. Es ist kein Wunder, daß den Astronomen bei so viel Arbeit in dieser Bergeseinsamkeit die Zeit nicht lang wird; zudem sind selbstredend auch hübsche Astronominnen dabei. 
Nachdem ich noch abends den Mars durch das große Fernrohr gesehen hatte, groß und leuchtend, fast wie die Mondscheibe, ging es in der Nacht wieder zu Tal. Merkwürdig war dabei die Abgrenzung des Nebels. Wir hatten den Sternenhimmel über uns und unter uns wie eine ebene Meeresfläche den Nebel. Mit einem Ruck kam der Wagen in den Nebel, die Sterne waren verschwunden und das Licht der Wagenlaterne drang nur noch wenige Schritte nach vorwärts.

Den nächsten Tag (Sonntag) benutzte ich, um mir San José anzusehen, kehrte aber schon zeitig nachmittags zurück, um zum Schlaf und zur Vorbereitung für die Vorlesung reichlich Muße zu haben.

Nicht weniger des Interessanten bot mein nächster Sonntagsausflug, der nach den Seebädern Monterey, Pacific-Grove und Santa Cruz am stillen Ozean ging. Ich war schon einmal von San Franzisko aus soweit hinausgefahren, daß ich den Ausblick auf den Großen Ozean voll genießen konnte; aber jetzt hatte ich Gelegenheit, in weiter Ausdehnung die Felsenufer und das Wellenspiel dieses Meeres zu bewundern. Aber weit mehr als alles dieses, interessierte mich ein kleines Häuschen in Pacific-Grove, wo Professor Löb sein Laboratorium hat.

Wie groß ist doch der Unterschied zwischen den Riesenwerken der Industrie und den Werkstätten der Wissenschaft! Welch imponierende Kolosse sind die Ozeandampfer! Aber wenn man öfter fährt, wird es klar, daß die Seeoffiziere, die Maschinisten und Matrosen immer den gleichen Dienst tun. In den Passagierräumen sprechen immer die gleichen Menschen über die gleichen Dinge, dehnen sich auf den gleichen Sesseln, werfen auf dem Oberdeck mit denselben Scheiben nach denselben Zielen. Riesige Massen, aber kein neuer Gedanke! In der Wissenschaft wurde allerdings auch schon manches durch Massenentwicklung geleistet (wir sahen es an der Licksternwarte); aber das wahrhaft Große (freilich unser Unterrichtsminister darf das nicht hören), wird immer mit den kleinsten Mitteln hervorgebracht.

Es muß groß sein, über Millionen zu verfügen zum Besten eines großen Volkes, an der Spitze von Hunderttausenden Schlachten zu gewinnen. Noch größer aber dünkt es mich, im bescheidensten Raume mit den bescheidensten Mitteln Wahrheiten zu entdecken, die noch das Fundament unseres Wissens bleiben werden, wenn die Erinnerung an jene Schlachten nur mehr mühsam in der Geschichte aufbewahrt wird. Was erhielt sich vom ganzen griechischen und römischen Volke heute noch in voller Lebensfrische und wirkt reicher und mächtiger fort als je? Die Marathonkämpfer wurden bei Vionville und Liaojang überboten. Die Leute, die zu ihrem Vergnügen Homer oder Sophokles lesen, sterben aus; aber der pythagoreische Lehrsatz, das archimedische Prinzip sind wirklich unsterblich.

Das sind meine allgemeinen Ansichten; wie weit sie auf Pacific-Grove an- 
wendbar sind, wird erst die Zukunft zeigen. Die dort gemachte Entdeckung brachte mich schon vor Jahren, als sie noch ganz neu war, in große Verlegenheit. Voll Feuereifer setzte ich sie in einer Gesellschaft auseinander, nicht ahnend, daß etwas so rein Sachliches, das so gar nicht auf die Absicht, Wollustgefühle zu erregen, ausging, ja gar nicht dazu fähig ist, unanständig sein könne. Erst der plötzliche, etwas auffällige Abgang meiner Tischnachbarin ließ mich das ahnen. Später sang dieselbe Dame ein sehr zweifelhaftes Lied von Aletter. Ich konnte mich der Bemerkung nicht enthalten, daß ich mich wundere, daß man dies für anständig, mein Thema aber für unanständig halte. Ja, Ihr Thema verstehen wir nicht, sagte die Dame und unwillkürlich antwortete ich: „Den Aletter aber verstehen Sie.“ Es ist das eine unserer alten Heucheleien, der die Temperenzler nun noch eine neue beifügen wollen. Ich werde mich gehörig winden müssen, um das Objekt der Löbschen Forschung klar zu machen, ohne Anstoß zu erregen.

Man glaubte lange, daß alle chemischen Verbindungen, welche für die lebenden Organismen charakteristisch sind, die sogenannten organischen Verbindungen, nur durch eine besondere Kraft, die Lebenskraft, erzeugt werden können. Heute weiß man, daß sich zahlreiche organische Verbindungen aus ihren chemischen Elementen durch gewöhnliche chemische Reaktionen ohne jede Spur von Lebenskraft zusammensetzen lassen. Aber viele glauben doch, daß das Leben selbst etwas ganz Besonderes, total von den begleitenden chemischen Prozessen verschiedenes sei und daß die speziellen Wirkungen des Lebens nie durch unbelebtes hervorgerufen werden können. Diese Ansicht ist durch die Forschungen Löbs zwar lange noch nicht widerlegt, aber es ist doch wieder ein neues Gewicht zu ihren Ungunsten in die Wagschale geworfen worden.

Es gibt bekanntlich Tiergattungen, bei denen sich unter Umständen das Ei des weiblichen Individuums ohne alle Befruchtung entwickeln kann. (Parthenogenesis.) Löb arbeitete nun mit Tiergattungen, wo dies niemals vorkommt, mit Seeigeln und Seesternen und zeigte, daß auf deren Eier die gleichen Wirkungen, die sonst nur der männliche Samen erzeugt, durch vollkommen leblose Säuren ausgeübt werden können, so daß sich die der Wirkung von Kohlensäure, Buttersäure oder Propylessigsäure unter passenden Umständen ausgesetzten Eier gerade so entwickeln, wie normal befruchtete.

Man begreift, wie wichtig die Entdeckung ist, daß sich ein Prozeß, der bisher nur als Folge einer besonderen Lebenstätigkeit angesehen wurde, auch durch rein chemische Reagentien herbeiführen läßt, und wenn dasselbe nicht bloß für die Seeigel, sondern auch für höhere Lebewesen bis zum Menschen hinauf gilt, welche sozialen Umwälzungen werden daraus folgen! Eine Frauenemanzipation, wie sie die heutigen Frauenrechtlerinnen nicht einmal träumen. Der Mann wird einfach überflüssig; ein Fläschchen, mit geschickt gemisch- 
ten Chemikalien gefüllt, ersetzt ihn vollständig. Dabei kann noch die Vererbung viel rationeller betrieben werden, als jetzt, wo sie so vielen Zufälligkeiten unterworfen ist. Nicht lange und man findet, welche Mischung Knaben, welche Mädchen liefert, und da die ersteren vollkommen überflüssig sind, werden davon nur wenige Exemplare für die zoologischen Gärten erzeugt. Dann freilich wird auch der Wein überflüssig sein.

Von Pacific-Grove fuhr ich nach dem Seebade Santa Cruz, welches besonders dadurch bemerkenswert ist, daß eine große Zahl von Badegästen nicht in Häusern, sondern in Leinwandzelten mit kleinen Leinwandfenstern wohnt, die wie Landwohnungen vermietet werden. Andere wohnen in kleinen Holzhäusern, welche auf Schiffen erbaut sind und in den seichten Buchten und ins Meer mündenden Flüssen bald da, bald dort hin gerudert werden können.

Überhaupt ist allenthalben die Kleinheit der fast ausschließlich aus Holz erbauten Häuser überraschend. In Berkeley gibt es viele Häuser, die lebhaft an das des Schnitzelbauern in der Umgegend von Graz erinnern, das sich dieser auf seinem kleinen Grunde selbst aus Holzbrettern gezimmert und geschnitzt hatte.

Dann sah ich mir noch die sogenannten big-trees an, deren Alter nach Jahrtausenden zählt. Ein allerdings nicht mehr lebender Stamm wird gezeigt, aus dessen Ringen man genau erkennen kann, wie viel Jahrtausende alt er wurde. Ich habe es wieder vergessen.

Die übrigen Wochentage außer Samstag und Sonntag waren der Arbeit gewidmet, aber doch auch keineswegs ohne jede Unterhaltung. Es gab viele Gesellschaften, darunter auch ein paar ganz feierliche. Bei einer derselben hatte mich mein Kollege, der mich abholen wollte, schon vorher mit echt englischer Sorgsamkeit für die Toilette ermahnt, evening-dress anzulegen. Als er eintrat, rief ich ihm entgegen: Bin ich nicht schön? Aber nein, o weh! Ich hatte das Stiefelputzen vergessen. Doch mein Kollege wußte Bescheid. Er führte mich in einen Raum im Souterrain, legte Rock, Weste und Manschetten $a b$, fand die erforderlichen Utensilien in einem Schranke und putzte mir eigenhändig mit Virtuosität die Stiefel. Dann trank er noch aus dem Glase, aus dem er früher Wasser auf die Wichse geträufelt hatte. Amerikanisch!

Auch Damen fehlten im geselligen Kreise nicht. Den Frauen der BerkeleyProfessoren schlossen sich solche der Zugereisten an, ferner die Wirtin mit ihren liebenswürdigen Töchtern, von denen eine reizend sang, und andere Freundinnen. Unter solchen Verhältnissen werde ich öfters und wurde damals von einer noch nicht aufgezählten Krankheit, der Dichteritis, befallen. Da ich es schon bei den ändern Krankheiten tat, so suche ich auch von ihrem Grade hier einen Begriff zu geben und lasse eines ihrer Produkte hier folgen:

An meine Frau. 
Soll ich mit fremden Fraun in der Ferne mich nicht unterhalten?

Sind sie von Allem, was hier, dir denn am ähnlichsten nicht?

Oft schon küßt' ich dein Bild auf Pappe: Oh so verzeihe,

Wenn ich dein Bild auch geküßt, fand ichs in Fleisch und in Blut!

Zudem bin Theoretiker ich von der Zeh' bis zum Scheitel

Und so vertrau', daß ich auch nur theoretisch geküßt.

Also Verzeihung von allen Seiten und auch wegen des Küssens. Es war nichts als eine poetische Notwendigkeit. Den möchte ich sehen, der mit bloßem Zusammenspazierengehen, Konversieren, Tennisspielen und Musizieren ein Gedicht fertig bringt!

Die Frauen sind übrigens in Kalifornien auffallend groß und stark gebaut und da auch die Bartentwicklung manchesmal nichts zu wünschen übrig läßt, mußte ich einem Kollegen recht geben, als er sagte: Finden Sie nicht, daß die Frauen in Amerika etwas Männliches haben? Dagegen wollte er mir nicht recht geben, als ich entgegnete: Und die Männer etwas Weibisches. Letzteres gilt übrigens nur bezüglich ihrer Bartlosigkeit; an Willensstärke, Mut, Unternehmungsgeist und Charakterfestigkeit stellen sie ihren Mann.

$\mathrm{Zu}$ den Dingen, die an Wochentagen Abwechslung brachten, gehörte auch der Besuch des amerikanischen Kriegsministers, der über San Franzisko nach den Philippinen ging. In seiner Begleitung soll Miß Roosevelt gewesen sein, sie kam mir aber nicht zu Gesicht. Der Kriegsminister besuchte eine Volksversammlung im großen Life-oak-Walde der Universität Berkeley. Da hätte man die naive Derbheit, Unternehmungslust und Begeisterung der Reden hören sollen! Nur ein Muster. Der Major von Berkeley stellte den Kriegsminister nach einer kurzen Ansprache mit folgenden Worten vor: Das ist Mr. Taft! ein guter Kriegsminister, ein guter Bürger und überhaupt in jeder Hinsicht ein guter, alter Kerl. Im Englischen klingt es noch vertraulicher: A good old fellow.

Ja, Amerika wird noch Großes leisten, ich glaube an dieses Volk, obwohl ich es bei einer Beschäftigung sah, die ihm am wenigsten gut lag, im theoretisch-physikalischen Seminare beim Integrieren und Differenzieren. Da schickten sie sich ungefähr so an, wie ich beim Springen über die Gräben oder Herablaufen über die Hügel, die man auf dem Grunde der BerkeleyUniversität so zahlreich passieren muß.

Endlich kam der Abend, wo ich zum letzten Male dem eintönigen Gesange der bebrillten Kellnerinnen lauschte. Als ich die letzte Omelette zerschnitten hatte, überblickte mein Kollege neben mir mit Falkenblick die Anzahl der Stücke und sagte: Zu jedem Stück ist noch eine halbe Minute Zeit. Dann packte mich die Eisenbahn und riß mich fort, zuerst nach Portland (zwei Nächte 
im Zug). Obwohl dort eine Ausstellung ${ }^{3}$ lockte, fuhr ich sofort durch nach Livingstone (wieder zwei Nächte im Wagen). Die Fahrt war wunderschön, wenn es nur immer Tag gewesen wäre! Das Herrlichste ist der Mount Shasta mit seinem hohen schneebedeckten Haupte in der subtropischen Vegetation. An manchem See fuhr ich vorüber, bergumragt, waldumkränzt, gegen den der Gmundner- und Attersee unbedeutend erscheinen. Hier sieht man kein Haus an dessen Ufer, ich weiß nicht einmal, ob alle schon Namen haben. Über den Yellowstonepark sage ich nichts. Er ist ein Wunder, wie es schwerlich in der Welt noch irgendwo existiert. Man lese das im Baedeker nach oder betrachte gute Bilder davon oder am besten, man sehe es sich in der Natur an, wenn man ausreichend Zeit, Geld und guten Humor hat. Aber man mache es nicht so wie ich. Man gehe anfangs Juni hin, wo die Hitze noch nicht so groß ist, und widme dazu 14 Tage oder besser einen Monat, daß man alles mit Muße ansehen kann und vom Staunen auch zum Genießen kommt.

Ich hatte mir zuviel des Guten aufgeladen. Jetzt sollte ich wieder vier Nächte in der Eisenbahn zubringen und war mit meiner Genußfähigkeit ebenso, wie mit meiner Wäsche zu Ende. Dazu die fürchterliche Hitze. Ich hatte immer ein Handtuch in der Hand, die man glücklicherweise in den amerikanischen Bahnen in beliebiger Menge bekommt, um mir den Schweiß abzuwischen. Ich begreife jetzt, was ein Schweißtuch ist. Zudem lieben es die Amerikaner, ihre Eisenbahnwagen hermetisch zu verschließen, nicht aus Furcht vor dem Luftzug, die sie nicht kennen, sondern vor dem Ruß. Die schönen Aussichtswagen ganz hinten, wo weniger Ruß ist, gibt es auf dieser Strecke nicht. Ich ließ einmal in meinem Abteil, das mehr vorne war, längere Zeit das Fenster offen, wurde aber dann so schwarz, daß ich mich nicht wundern würde, wenn im künftigen Jahrhundert ein Gelehrter einmal die Hypothese aufstellte, die Neger seien dadurch so schwarz geworden, daß sie immer als Eisenbahnpersonal benutzt wurden.

Nun kehrte auch noch mein Magenkatarrh wieder. Man bekommt in dem Speisewagen zwar Wein, aber nur ungern; erst nach dem Essen, wenn die meisten Gäste, besonders die Damen, schon fortgegangen sind. Das erste, was man bekommt, ist ein Glas Eiswasser und ein Zettel, auf den man gleich alles, was man will, auf einmal aufschreiben muß. Nun dauert es eine Ewigkeit, bis nur der Zettel abgeholt wird und man sitzt mit vor Hitze vertrocknetem Gaumen immer neben dem Eiswasser. Ich unterlag der Versuchung (Engel wären erlegen) und trank von dem Gift.

Ja auf einmal bekam ich gar keinen Wein mehr. Das klärte sich so auf: Der

\footnotetext{
${ }^{3}$ Lewis and Clark Exposition

Meriwether Lewis und William Clark erkundeten 1804 bis 1806 im Auftrag des Präsidenten Jefferson das Gebiet des heutigen Bundesstaates Oregon. Am 7. 11. 1805 erreichten sie den Pazifik.
} 
ganze Staat Nord-Dakota ist abstinent und während der Zug durch denselben fährt, darf kein Wein verzapft werden. Ich protestierte: Was geht mich der Staat Nord-Dakota an! Ich will nichts weiter als nach Wien kommen. Führt mich meinetwegen durch das Land, wo der Pfeffer wächst! O, Pfeffer wird hier sehr viel angebaut, antwortete man mir. Zum Teufel! hier werden selbst unsere kräftigsten Flüche zuschanden. Freilich gegen Trinkgeld und noch mehr versteckt bekam ich doch Wein; aber er mußte unter der Hand bezahlt werden und durfte nicht auf der bill of fare stehen.

Die amerikanischen Bahnverwaltungen hängen gewöhnlich erst einen neuen Wagen an, wenn die vorhandenen gefüllt sind, und trotzdem haben die Züge enorme Länge. Die Einzelwagen haben Namen wie die Schiffe, sonst würde man sich gar nicht mehr zurechtfinden. Ich fuhr der Reihe nach in den Wagen Sant Jesabel, Pembina und Vernedal. Sie wimmeln von Publikum von allen Nationalitäten und Menschenrassen, die bei der großen Hitze oft etwas degagiert angekleidet sind. Ein Säugling lag immer vollkommen nackt auf dem Sammetfauteuil und erinnerte mich an die Bilder vom Christuskinde, das freilich in keinem Pullmancar lag. Ich wollte dies der Mutter als Schmeichelei sagen, aber wie unschön klingt alles im Englischen: äs de tscheild tschises kreist!

Es ist ein Glück, daß ich nicht als Engländer geboren wurde. Ich hätte nie eine Braut heimgeführt. Man kann sich denken, daß ich bei der Liebeserklärung etwas verlegen war. „Der schaut drein, als müßt’ er in den Hörsaal hinein" sagt Mephisto. Ja, wenn ich nur nichts weiter hätte müssen, als in den Hörsaal hinein, wenn nichts weiter als Frau Physik und Metaphysika vor mir gestanden wären! Aber vor mir stand ein liebliches, junges Mädchen. Da ging es schwer mit der Rede; doch als ich zum punktum saliens kam, da half mir die Weisheit und der gute Geschmack unserer Ururahnen, die für das höchste der Gefühle auch das wohlklingendste Wort gefunden haben, das Wort Liebe. Wie am Steinwayflügel die Musik, so regierte jetzt nicht ich die Sprache, sondern die Sprache riß mich hin und ich erzielte einen vollen Erfolg. Aber wenn ich in echt englischer Aussprache hätte sagen müssen: „Ei lowff ju“, meine Auserkorene wäre davongelaufen, wie die Hühner vor dem Atemholen des sie lockenden kropfigen Steirers.

Die ganze bunte Menge der Fahrgäste muß nun abends in die Betten gedrängt werden. Die Einrichtung der amerikanischen Schlafwagen ist folgende: Durch die Mitte des ganzen Wagens läuft ein ziemlich schmaler Gang. Zu beiden Seiten sind gepolsterte Bänke, auf deren jeder zwei Personen sitzen können. Jeder Fahrgast bekommt eine solche Bank zugewiesen. Abends wird der Raum über je zwei auf derselben Seite des Ganges liegenden Bänken, die immer gegeneinander schauen, in zwei übereinanderliegende Betten verwandelt, die durch herabhängende Vorhänge gegen den Gang abgeschlossen sind. 
Die Längsrichtung der Betten ist parallel der Fahrtrichtung. Man kann wohl auch zwei übereinanderliegende Betten für sich haben - ein ganzes Kompartiment — das kostet aber das Doppelte. Da man keine Schublade hat, so muß man Nachtwäsche, Pantoffel und was man sonst braucht, in die Handtasche packen, die dann der Mohr ins Bett stellt. Dann hat man sich hinter dem Vorhange auszukleiden, im Bette selbst aber die Kleider und Handtasche unterzubringen und dann zu schlafen, ohne zu ersticken.

Jeder Raum hat nur eine kleine, durch ein feines Gitter bedeckte Öffnung, die ins Freie führt und wird bei heißem Wetter so dunstig, daß ich im Kostüme jenes Säuglings schlief, wodurch ich auch die Mühe des Auspackens der Wäsche ersparte. Einmal ließ ich ein ganzes Fenster bei Nacht offen, da sagte aber dann nach dem Aufstehen der Mohr zu mir: Herr Kollege.

Um Uhr, Geldtasche, Augengläser usw. nicht zu verlieren, gab ich in das weitmaschige Netz, den einzigen Aufbewahrungsort im ganzen Bettraume, zuerst den Hut und in diesen dann all die Kleinigkeiten. Der Mohr aber hängte den Hut beim Bettmachen immer oben in unerreichbarer Höhe auf und es war komisch, wie perplex er darüber war, daß ich im Bette den Hut brauchte.

Die kritischste Zeit ist nun die der Umwandlung der Bänke in die Betten. Man kann in den Bänken nicht mehr sitzen und auch das Bett ist noch nicht fertig. Ich flüchte mich in einen Waschraum; allein da bürstet ein Passagier Wolken Staubes aus seinen Kleidern, ein anderer spritzt beim Waschen Wasser nach allen Windrichtungen. Ich versuche den Salonwagen zu erreichen, der übrigens bei vielen Zügen fehlt; allein da müßte ich die Gänge von sieben bis acht Wagen passieren, alle mit Bettvorhängen zu beiden Seiten abgeschlossen, diese sind lebendig; aus ihrer Verborgenheit versetzt uns bald ein Arm, bald ein Fuß, bald ein weicherer Gegenstand einen Stoß. Zudem stolpert man fortwährend über die unten vorstehenden Gepäckstücke. Endlich finde ich Platz in einem Kompartiment, das noch unversehrte Bänke hat. Auf der anderen Seite des Ganges ist schon ein Bett, dessen Vorhang in steter Bewegung begriffen ist. Es hat doch etwas auf sich mit dem ewig Weiblichen. Beim Indextestieren in der Wiener Vorlesung sehe ich immer nur die Hände der Studenten, aber ich erkenne sofort jede Frauenhand. So war ich auch überzeugt, daß dieser Vorhang weibliche Glieder birgt und bald lüftete er sich auch, durch eine unvorsichtige Bewegung beim Auskleiden und ich sah, daß ich recht hatte.

Vielen Unbequemlichkeiten der Morgenstunden entging ich durch meine Gewohnheit, zeitlich aufzustehen. So war ich beim Anziehen und Waschen allein und rief den später sich im Waschraume Drängenden die schon von Bismarck zitierten Worte aus Mozarts Entführung zu „Mich zu hintergeh'n, müßt ihr früh aufsteh'n" 
Ich war durch die Hitze und den Ruß, durch Magenkatarrh und Durst schließlich so reisemüde geworden, daß ich nicht nur auf die Sonnenfinsternis verzichtete, sondern noch den Anschluß an den Kaiser Wilhelm II. zu gewinnen suchte, welcher mich am raschesten heimbrachte. Nun war aber gerade ein Streik der Telegraphisten ausgebrochen, infolgedessen wir sechs Stunden Verspätung hatten. Ich geriet darüber in Wut; aber da sollte man das Phlegma der Amerikaner sehen! Sie blicken den Schimpfenden fast mitleidig an, als ob sie sagen wollten, der gute Mann meint, das hülfe etwas. In meinem Falle sagte der Kondukteur kurz: Wir wollen keinen Zusammenstoß riskieren.

In Chicago hatte ich nur zwölf Minuten Zeit und sollte noch von der Canalstreet Union Station zur Nickelplate-Station gelangen. Mit Gepäck beladen eilte ich planlos hin und her. Zwei, die ich um Auskunft gebeten hatte, hatten nicht geantwortet. Da bemerkt dies eine junge Dame und fragt mich zuvorkommend, was ich wünsche. Sie kann mir freilich auch keine Auskunft erteilen, vielleicht verstand sie nicht einmal meine Frage, aber sie zeigt mir einen Schutzmann, den ich in meiner Aufregung, so groß er war, nicht bemerkt hatte. Als ich ihr nicht aus Schmeichelei, sondern aus vollem Herzen zurief: „You are an Angel“, da seh' ich erst, es war Zug für Zug der Schutzengel vom pozzo di Verona. Sollte der Schutzengelglaube kein bloßes Märchen sein? Und wie paßt Märchenstimmung in die Hallen der Canalstreet Union Station in Chicago? Also fort vom Schutzengel zum Schutzmann, der mir rasch die gewünschte Auskunft erteilte, so daß ich noch rechtzeitig zum andern Bahnhof kam.

In New-York hatte ich noch eine Überraschung. Der schöne Pier, der von der Eisenbahn zur Überfuhr geführt hatte, war abgebrannt, und ich mußte, immer mit meinem Gepäck beladen, über verkohlte Holzstücke stolpern.

Trotz aller Hindernisse erreichte ich den Kaiser Wilhelm II. noch rechtzeitig. Als ich samt allem großen und kleinen Gepäck an Bord war, wie schlug da mein Herz freudig!

„Stimmet an die frohen Lieder,

Denn dem väterlichen Herd

Ist das Schiff nun zugekehrt

Und zur Heimat geht es wieder."

Die Rückfahrt war von dem herrlichsten Wetter begünstigt. Die gute Schiffskost stellte auch meinen Magen wieder vollständig her. Ich trank keinen Tropfen Wasser, auch nicht viel Bier, aber desto mehr edlen Rüdesheimer. Auf dem Schiffe ist das so günstig; wenn man ein wenig wackelt, schreibens alle der Schiffsbewegung zu.

Nun noch die winzige Eisenbahnfahrt von Bremen bis Wien, eine fesche Fahrt in einem Wiener Fiaker und ich bin zu Hause. Ja so eine Reise hat viel 
Interessantes und Großartiges, Kalifornien ist schön, der Mount Shasta ist herrlich, der Yellowstonepark wundervoll; aber weitaus das Schönste an der ganzen Reise, das ist doch der Moment, wo man wieder daheim ist. 


\section{Personenregister}

Das Personenregister enthält alle in Boltzmanns Reisebericht genannten Personen. Die Kurztexte sind nach Möglichkeit aus dem Brockhaus entnommen; bei nur beiläufig erwähnten berühmten Personen beschränken sich die Angaben auf die Lebensdaten und den Beruf.

Die Literaturquellen zerfallen in drei Gruppen:

- Lexika

Neben den eigentlichen Lexika sind hier auch Beiträge in Biographiesammlungen angegeben.

- Biographie

Die angegebenen Biographien sind in Heidelberger Bibliotheken verfügbar. Soweit sie im Besitz der Univ. Bibliothek sind, ist die Standort-Signatur angegeben; bei anderen Bibliotheken ist lediglich die Bibliothek benannt.

- Internet

Nach Möglichkeit wurden Permanenz versprechende Internetseiten ausgewählt.

Im Anhang sind die verwendeten Lexika mit der Signatur der Univ. Bibliothek verzeichnet.

Aletter, Wilhelm, ${ }^{*}$ 27. 1. 1867, $†$ 30. 6. 1934, Pianist und Komponist.

Internet:

www.dolmetsch.com/cdefsa1.htm

Amati, ital. Geigenbauerfamilie in Cremona im 16. und 17. Jh., deren Instrumente sich durch eine zarte, helle Färbung des Tons auszeichnen.

Lexika: Brockhaus 1, S. 484

Barth, Karl Heinrich, * Pillau 12. 7. 1847, † Berlin 23. 12. 1922, Klavierpädagoge.

Internet:

de.wikipedia.org/wiki/Karl_Heinrich_Barth

Beethoven, Ludwig van, Komponist, * Bonn 1770, † Wien 26. 3. 1827. 


\section{Lexika:}

Brockhaus 3, S. 15-17

\section{Biographie:}

Geck, Martin: Ludwig van Beethoven / dargestellt von Martin Geck. Reinbek bei Hamburg : Rowohlt, 1996. - 157 S. : Ill., Notenbeisp.

ISBN 3-499-50570-3

UB-Signatur: 96 A 4364

Kinderman, William: Beethoven / William Kinderman. - Oxford [u.a.]

: Oxford University Press, 1995. - XVI, 374 S. : Ill., Notenbeisp. ISBN 0-19-816521-8

UB-Signatur: 96 A 10872

\section{Internet:}

de.wikipedia.org/wiki/Ludwig_van_Beethoven

Berkeley, George, irischer Philosoph und Theologe, * Disert Castle (Cty. Kilkenny) 12. 3. 1685, † Oxford 14.1.1753; versuchte 1729-31 ein Missionszentrum auf den Bermudainseln einzurichten. Nach seiner Rückkehr wurde B. 1734 zum Bischof von Cloyne (bei Cork) ernannt. - Seine Lehre bildete sich in krit. Auseinandersetzung mit R. DESCARTES und J. LOCKE. Anregungen empfing B. von N. MALEBRANCHE. B. lehnte den Materialismus, unter dem er die Annahme einer außerhalb des Bewußtseins existierenden Körperwelt verstand, ab. Nicht nur die sekundären Sinnesqualitäten wie Farben, Töne sind für ihn, wie schon für LOCKE, subjektiv, sondern darüber hinaus ebenfalls die primären Qualitäten wie Ausdehnung und Bewegung. Sinnl. Gegenstände sind immer als Vorstellungen oder Ideen (>ideas<) gegeben; ihr Sein besteht daher in ihrem Wahrgenommenwerden (>esse est percipi<) oder ihrem Wahrgenommenwerdenkönnen (wenn nicht aktual gegeben). Gegen die irrtümlich als subjektiven Idealismus gedeutete Lehre wandte sich später I. KANT. B. stellte die Außenwelt nicht grundsätzlich in Frage. Neben den Ideen de Einbildungskraft, die dem menschl. Willen entstammen, gibt es Ideen, die eine vom Bewußtsein unabhängige, als naturgesetzlich erfahrene Ordnung aufweisen. Den Grund dieser Wahrnehmungswelt sieht B. in einer äußeren Ursache, in Gott als einem denkenden Geist, in dem die Ideen existieren, solange sie nicht aktual vom Menschen gedacht werden, und der sie im Wahrnehmenden hervorruft. Bereits die erste bedeutende Schrift $>$ An essay towards a new theory of vision $<(1709$; dt. $>$ Versuch einer neuen Theorie der Gesichtswahrnehmung $<$ ) enthält die zentralen Teile einer Theorie der Wahrnehmung und des Wissens mit der B. die Tradition der angelsächs. Rationalismuskri- 
tik fortsetzt. Seinen Immaterialismus entwickelte B. in seinem Hauptwerk $>$ Treatise concerning the principles of human knowledge $<(1710$; dt. >Abhandlung über die Prinzipien menschl. Erkenntnis $<$ ) sowie in $>$ Three dialogues between Hylas and Hylonous< $(1713$; dt. $>$ Drei Dialoge zw. Hylas und Hylonous $<$ ) fort, sodann in seiner Schrift > Aleiphron or the minute philosopher $<$ (1732), einer Verteidigung des Christentums gegen die Freidenker, bes. gegen die Ethik A. SHAFTESBURYS. $>$ Siris< $(1744$; dt.) enthält, von einem Allheilmittel, dem Teerwasser, ausgehend neuplatonische metaphys. Gedanken.

(aus Brockhaus)

\section{Lexika:}

Brockhaus 3, S. 140

\section{Biographie:}

Berman, David: George Berkeley : idealism and the man / David Berman. - Oxford : Clarendon, 1994. - XI, 230 S. : Ill., graph. Darst.

ISBN 0-19-826746-0

UB-Signatur: 94 A 4730

Jessop, Thomas E.: A bibliography of George Berkeley / by T. E. Jessop. - The Hague : Nijhoff, 1973. - XX, 155 S.

ISBN 90-247-1577-6

UB-Signatur: IZA Pers-BERK 001

\section{Internet:}

www-history.mcs.st-andrews.ac.uk/Mathematicians/Berkeley

Bismarck, Otto (Eduard Leopold) von, Graf von B.-Schönhausen (seit 1865), Fürst von B. (seit 1871), Herzog von Lauenburg (seit 1890), Staatsmann, * Schönhausen 1.4.1815, † Friedrichsruh 30.7.1898,

\section{Lexika:}

Brockhaus 3, S. 388-389

DBE 1, S. 545

ADB Bd. 46 S. 571-775

Biographie: Gall, Lothar: Bismarck : der weisse Revolutionr / Lothar Gall. - Korrigierte Ausg. - Frankfurt/M [u.a.] : Ullstein, 1993. - 812 S.

: Ill. - (Ullstein-Buch ; Nr. 23286 : Sachbuch)

ISBN 3-548-23286-8

UB-Signatur: LSA His-OQ 014

\section{Internet:}

de.wikipedia.org/wiki/Otto_von_Bismarck

www.preussen-chronik.de/person.jsp?key=Person_Otto + Eduard + Leopold + von_Bismarck 
Böcklin, Arnold, schweizer. Maler, * Basel 16. 10. 1827, † San Domenico (bei Fiesole) 16. 1. 1901.

Lexika: Brockhaus 3, S. 478

\section{Biographie:}

Böcklin, Arnold: Leben und Werk in Daten und Bildern / Arnold Boecklin. [Hrsg.] Lutz Tittel. - Frankfurt a. M. : Insel-Verl., 1977. - 196 S. : Ill.

ISBN 3-458-31984-0

UB-Signatur: 77 A 2463

\section{Internet:}

de.wikipedia.org/wiki/Arnold_B\%C3\%B6clin

Bösendorfer, Ignaz, österr. Klavierbauer, * Wien 27. 7. 1794, † ebd. 14. 4. 1859; gründete 1828 eine Klavierfabrik in Wien, bis heute eine der führenden Weltfirmen. B.-Instrumente zeichnen sich durch einen bes. $>$ kantablen $<$ Klang aus.

Lexika: Brockhaus 3, S. 586

\section{Internet:}

www.luxurypianos.com/history_of_grands.htm

Boltzmann, Ludwig, österr. Physiker, * Wien 20.2.1844, † (Selbstmord) Duino 5.9.1906; ab 1869 Prof. an versch. österr. und dt. Univ., zuletzt (1902) in Wien. B. bestätigte 1872 die damals noch umstrittene maxwellsche Elektrodynamik, indem er den von J.C. Maxwell geforderten Zusammenhang zw. opt. Brechzahl und Dielektrizitätskonstante experimentell bei Schwefel nachwies. Sein Hauptarbeitsgebiet war aber die theoret. Physik.

...

(aus Brockhaus)

\section{Lexika:}

Brockhaus 3, S. 536-537

DBE 2, S. 12-13

DSB 2, S. 260-268

NDB Bd. 2, S. 436 f.

Pogg. III. S. 155-156, IV. S. 153

\section{Biographie:}

Broda, Engelbert: Ludwig Boltzmann : Mensch, Physiker, Philosoph / 
Engelbert Broda. Neu hrsg. von Gitta Deutsch .... - Wien : Deuticke, 1986. - 149 S. : Ill.

ISBN 3-7005-4560-6

UB-Signatur: 89 H 226

Ludwig Boltzmann : his later life and philosophy ; 1900 - 1906 / ed. by John Blackmore. - Dordrecht : Kluwer

Bd. 1. 1995

Bd. 2. 1995

UB-Signatur: 95 H 417

Stiller, Wolfgang: Ludwig Boltzmann : Altmeister der klassischen Physik, Wegbereiter der Quantenphysik und Evolutionstheorie / von Wolfgang Stiller. - Thun [u.a.] : Deutsch, 1989. - 212 S. : Ill.

ISBN 3-8171-1115-0

UB-Signatur: 89 H 1553

\section{Internet:}

www.ub.uni-hd.de/helios/fachinfo/www/math/homo-heid/boltzmann.htm www-history.mcs.st-and.ac.uk/history/Mathematicians/Boltzmann.html de.wikipedia.org/wiki/Ludwig_Boltzmann

Campbell, William Wallace, amerikan. Astronom, * in der Hancock Cty. (Oh.) 11.4. 1862, † San Francisco 14.6. 1938; seit 1901 Prof. am LickObservatorium in Kalifornien; wirkte bahnbrechend auf dem Gebiet der Vermessung von Sternspektren und der Bestimmung der Radialgeschwindigkeiten von Fixsternen.

(aus Brockhaus)

\section{Lexika:}

Brockhaus 4, S. 284

\section{Internet:}

www.phys-astro.sonoma.edu/BruceMedalists/Campbell/

Clark, Alvan Graham, amerikan. Optiker und Astronom, * Fall River (Mass.) 10. 7. 1832, † Cambridge (Mass.) 9.6. 1897; fertigte wie sein Vater (A. Clark, $\dagger 1804, \dagger 1887)$ große Refraktoren und die benötigten Linsen für zahlreiche amerikan. und europ. Sternwarten (u.a. 1897 das Objektiv für den großen Yerkes-Refraktor, der mit einer Öffnung von $102 \mathrm{~cm}$ bis heute das größte Linsenfernrohr der Erde blieb). Bei seinen astronom. Beobachtungen entdeckte er 16 Doppelsterne, u.a. 1862 mit seinem Vater den Begleiter des Sirius.

(aus Brockhaus) 


\section{Lexika:}

Brockhaus 4, S. 590

\section{Biographie:}

Warner, Deborah Jean: Alvan Clark \& Sons : Artists in optics / Debohrah Jean Warner. - Washington : Smithsonian Inst. Pr., 1968. - 120

S. : Ill. - (United States National Museum : Bulletin ; 274)

UB-Signatur: 69 B 174

\section{Internet:}

www.infoplease.com/ce6/people/A0812411.html

Credner, (Karl) Hermann (Georg), Geologe und Paläontologe, * Gotha 1. 10. 1841, † Leipzig 22. 7. 1913; ab 1870 Prof. in Leipzig, ab 1872 Direktor der sächs. geolog. Landesanstalt. Er veröffentlichte zahlr. geolog. Karten von Sachsen, v. a. im Maßstab 1: 25000.

(aus Brockhaus)

\section{Lexika:}

Brockhaus 4, S. 726

\section{Internet:}

www.uni-leipzig.de/journal/gesichter/0406.html

Gutenberg, Johannes, eigtl. J. Gensfleisch zur Laden gen. G., Buchdrucker,

* Mainz zw. 1397 und 1400, † ebd. 3. 2. 1468; Erfinder des Buchdrucks mit bewegl. Metalllettern. ...

(aus Brockhaus)

\section{Lexika:}

Brockhaus 9, S. 306-307

\section{Biographie:}

Füssel, Stephan: Gutenberg und seine Wirkung / Stephan Füssel. Frankfurt am Main ; Leipzig : Insel-Verl., 1999. - 140 S., [32] Bl. : Ill. ISBN 3-458-16980-6

UB-Signatur: LSA HS-LD 007

\section{Internet:}

de.wikipedia.org/wiki/Johannes_Gutenberg

Hann, Julius (Ferdinand) Edler von (ab 1910), österr. Meteorologe, * Schloß Haus (bei Linz) 23. 3. 1839, † Wien 1. 10. 1921; ab 1874 Prof. in Wien (1897-1900 in Graz); bedeutendster Meteorologe und Klimatologe seiner Zeit. H. führte erstmals thermodynam. Prinzipien in die Meteorologie ein und wendete sie zur Erkärung der Wolken- und Niederschlagsbildung sowie des Föhns (1866) und bei der Untersuchung der Zyklonen 
und Antizyklonen sowie der atmosphär. Zirkulation an. H. entwickelte außerdem eine neue Theorie der Berg- und Talwinde. Grundlegend waren auch seine Arbeiten zur Klimatologie, für die er die erste umfassende Darstellung gab (>Hb. der Klimatologie<, 1883, 3 Bde. 1908-11), und sein $>$ Lb. der Meteorologie< (1901).

(aus Brockhaus)

\section{Lexika:}

Brockhaus 9, S. 472

\section{Internet:}

www.aeiou.at/aeiou.encyclop.h/h168041.htm

Haydn, (Franz) Joseph, österr. Komponist, * Rohrau 1732, † Wien 31. 5. $1809 . . .$.

(aus Brockhaus)

\section{Lexika:}

Brockhaus 9, S. 578-579

\section{Biographie:}

Knispel, Claudia Maria: Joseph Haydn / dargestellt von Claudia Maria Knispel. - Reinbek bei Hamburg : Rowohlt, 2003. - 159 S. : Ill., Notenbsp.

ISBN 3-499-50603-3

UB-Signatur: 2004 A 1315

Pieck, Werner: Haydn, der große Bassa : Biographie / Werner Pieck. Hamburg : Europäische Verlagsanstalt, 2004. - 258 S. : Ill.

ISBN 3-434-50501-6

UB-Signatur: 2004 A 2180

\section{Internet:}

de.wikipedia.org/wiki/Joseph_Haydn

Hearst, Phoebe Apperson, 1842-1919, Stifterin der Berkely University.

\section{Internet:}

www.hearstcastle.org/history/phoebe_hearst.asp

Helmholtz, Hermann (Ludwig Ferdinand) von (seit 1882), Naturforscher, * Potsdam 31. 8. 1821, † Charlottenburg 8. 9. 1894; Prof. der Physiologie in Königsberg (Pr.), Bonn und Heidelberg, seit 1871 Prof. der Physik in Berlin; ab 1888 erster Präsident der neu gegründeten PhysikalischTechn. Reichsanstalt in Charlottenburg.

(aus Brockhaus) 


\section{Lexika:}

Brockhaus 9, S. 681

HGL S. 108-109

ADB Bd. 51 S. 461-472

NDB Bd. 8, S. 498-501

Pagel Sp. 713-715

Pogg. I. Sp. 1059-1060, III. S. 611-612, IV. S. 612-613

\section{Biographie:}

Ebert, Hermann: Hermann von Helmholtz / von Hermann Ebert. Stuttgart : Wiss. Verlagsges., 1949. - 199 S. : Ill.

UB-Signatur: F 6834-7-12

Hermann von Helmholtz and the foundations of nineteenth-century science / ed. by David Cahan. - Berkeley [u.a.] : Univ. of California Pr., 1993. - XXIX, 666 S.

ISBN 0-520-08334-2

UB-Signatur: 94 H 600

\section{Internet:}

www.ub.uni-hd.de/helios/fachinfo/www/math/homo-heid/helmholtz.htm www-history.mcs.st-and.ac.uk/history/Mathematicians/Helmholtz.html

Homer, griech. Homeros, griech. Dichter, nach der Überlieferung ältester Dichter des Abendlandes; lebte im 8. Jh. v. Chr. im ion. Kleinasien.

(aus Brockhaus)

\section{Lexika:}

Brockhaus 10, S. 226-227

\section{Biographie:}

Schadewaldt, Wolfgang: Von Homers Welt und Werk : Aufsätze und Auslegungen zur homerischen Frage / Wolfgang Schadewaldt. - Leipzig : Koehler + Amelang, 1944. - 353 S. : Ill.

$$
\text { UB-Signatur: D 136-53 }
$$

\section{Internet:}

de.wikipedia.org/wiki/Homer

Ivanovici, Iosef, 1845-1902, Komponist. Komponierte die Donauwellen.

\section{Internet:}

www.johann-strauss.org.uk/composers/index.php3?content=ivanovici

Kirchhoff, Gustav (Robert), Physiker, * Königsberg (heute Kaliningrad) 12.3.1824, † Berlin 17.10.1887. Bereits als Student in Königsberg 
(1845/46) entdeckte K. die Gesetze der Stromverzweigung. 1850 wurde K. nach Breslau berufen, wo er im folgenden Jahr $R$. Bunsen kennen lernte, dem er 1854 nach Heidelberg folgte. Zus. mit diesem entwickelte K. die Spektralanalyse (1859/60). . . 1875-86 war K. Prof. in Berlin. (aus Brockhaus)

\section{Lexika:}

Brockhaus 12, S. 24

HGL S. 135

ADB Bd. 51 S. 165-167

NDB Bd. 11, S. 649-653

Pogg. I. Sp. 1260-1261, III. S. 720-721, IV. S. 750-751

\section{Biographie:}

Boltzmann, Ludwig: Gustav Robert Kirchhoff : Festrede zur Feier des 301. Gründungstages der Karl-Franzens- Universität zu Graz / gehalten am 15. November 1887 von Ludwig Boltzmann. - Leipzig : Barth, 1888. - 32 S. : Ill.

UB-Signatur: F 6970-1-50

\section{Internet:}

www.ub.uni-hd.de/helios/fachinfo/www/math/homo-heid/kirchhoff.htm www-history.mcs.st-and.ac.uk/history/Mathematicians/Kirchhoff.html

Klein, (Christian) Felix, Mathematiker, * Düsseldorf 25. 4. 1849, † Göttingen 22. 6. 1925; seit 1872 Prof. in Erlangen, 1875 in München, 1880 in Leipzig und ab 1886 in Göttingen. K.s Arbeitsgebiete waren v. a. die Funktionentheorie und die Geometrie. Sein 1872 vorgelegtes Erlanger Programm schuf, gestützt auf den bei seinem Lehrer C. JORDAN entlehnten Gruppenbegriff, Ordnung in der Vielzahl der Geometrien. 1871 und 1873 publizierte K. zwei wichtige Arbeiten zur nichteuklid. Geometrie, in denen er das heute nach ihm benannte projektive Modell derselben entwickelte. Die nachfolgenden Jahre galten der Auflösung der Gleichung fünften Grades mit transzendenten Mitteln sowie der Entwicklung der Theorie der ellipt. und der automorphen Funktionen. In den 90er Jahren setzte sich K. verstärkt mit den Anwendungen der Mathematik auseinander und schrieb mit A. SOMMERFELD ein Werk über den Kreisel. Zunehmend wurde K. auch wissenschaftsorganisatorisch tätig. Nach 1900 setzte er sich für die Modernisierung des mathemat. Unterrichts an Schule und Hochschule ein. (aus Brockhaus)

\section{Lexika:}

Brockhaus 12, S. 73 
Meschkowski S. 140-144

DMV Bd. 34, S. 89 und 197-213

NDB Bd. 11, S. 736 f.

Pogg. III. S. 724-725, IV. S. 756-757

\section{Biographie:}

Tobies, Renate: Felix Klein / Renate Tobies. - Leipzig : Teubner, 1981. - 104 S. : Ill.

Bereichsbibliothek Mathematik

\section{Internet:}

www-history.mcs.st-and.ac.uk/history/Mathematicians/Klein.html

Kolumbus, Columbus, Christoph, ital. Cristoforo Colombo, span. Cristobal Colon, genues. Seefahrer in span. Diensten, ${ }^{*}$ Genua 1451, $\dagger$ Valladolid 20. 5. 1506 .

\section{Lexika:}

Brockhaus 12, S. 209-210

\section{Biographie:}

Columbus : Lebensbilder / Siegfried Fischer-Fabian ; Karl-Heinz Jürgens. - Bergisch Gladbach : Lübbe, 1991. - 223 S. : zahlr. Ill. ISBN 3-7857-0605-7

\section{UB-Signatur: $91 \mathrm{~K} 264$}

Fernández-Armesto, Felipe: Columbus / Felipe Fernandez-Armesto. Oxford [u.a.] : Oxford University Press, 1991. - XX, 218 S. : Ill., Kt., graph. Darst.

ISBN 0-19-215898-8

UB-Signatur: 92 H 987

\section{Internet:}

de.wikipedia.org/wiki/Christoph_Kolumbus

Kronecker, Leopold, Mathematiker, * Liegnitz 7. 12. 1823, † Berlin 29. 12. 1891; seit 1861 Mitgl. der Preuß. Akademie der Wissenschaften in Berlin; seit 1883 Prof. an der Berliner Univ. K. leistete wichtige Beiträge zur Algebra und zur Arithmetik. K. lehnte die damals aufkommende mengentheoretisch orientierte Grundlegung der Analysis ab und übte u. a. heftige Kritik an G. CANTOR.

(aus Brockhaus)

\section{Lexika:}

Brockhaus 12, S. 569

Meschkowski S. 147-148 
DMV Bd. 2, S. 5-31

ADB Bd. 51 S. 393-395

NDB Bd. 13, S. 82 f.

Pogg. I. Sp. 1321, III. S. 752, IV. S. 807-808

\section{Internet:}

www-history.mcs.st-andrews.ac.uk/Mathematicians/Kronecker.html

Kummer, Ernst Eduard, Mathematiker, ${ }^{*}$ Sorau (Lausitz) 29.1.1810, $†$ Berlin 14.5. 1893; 1842 Prof. in Breslau, ab 1855 in Berlin. K. beschäftigte sich mit Problemen der Analysis (Integrationstheorie, hypergeometr. Reihe) und mit der Zahlentheorie. Ein weiterer Arbeitsschwerpunkt K.s war die Geometrie (u. a. Entdeckung der Kummerschen Fläche, einer Fläche vierter Ordnung).

(aus Brockhaus)

\section{Lexika:}

Brockhaus 12, S. 628

Meschkowski S. 149-150

DMV Bd. 3, S. 13-28

ADB Bd. 51 S. $438-440$

NDB Bd. 13, S. 282 f.

Pogg. I. Sp. 1329-1330, III. S. 757, IV. S. 817

\section{Biographie:}

Hensel, Kurt: Ernst Eduard Kummer und der grosse Fermatsche Satz : akademische Festrede zu Kaisers Geburtstag / gehalten von Kurt Hensel. - Marburg : Elwert, 1910. - 22 S.

UB-Signatur: ::23

\section{Internet:}

www-history.mcs.st-and.ac.uk/history/Mathematicians/Kummer.html

Lick, James, * Fredericksburg 25. 8. 1796, † 1. 10. 1877, amerikan. Geschäftsmann, Stifter des Lick-Observatoriums.

\section{Internet:}

www.atheists.org/Atheism/roots/lick/

en.wikipedia.org/wiki/James_Lick

Lick-Observatorium [nach dem Stifter, dem amerikan. Geschäftsmann J. Lick, $\left.{ }^{*} 1796, \dagger 1876\right]$, Sternwarte auf dem Mount Hamilton, USA. Hauptinstrument ist das 1959 in Betrieb genommene Spiegelteleskop mit einer freien Öffnung von $305 \mathrm{~cm}$ (Brennweite $1525 \mathrm{~cm}$ ). (aus Meyer) 


\section{Lexika:}

Meyer 15, S. 78

\section{Internet:}

www.snap-design.com/HVAG/lick/history/bal-2/2ed9.htm

Loeb, Jacques, 1859-1924, Physiologe.

\section{Biographie:}

Pauly, Philip J.: Controlling life : Jacques Loeb and the engineering ideal in biology / Philip J. Pauly. - New York, NY [u.a.] : Oxford Univ. Pr., 1987. - 252, [12] S. : Ill.

ISBN 0-19-504244-1

UB-Signatur: 88 H 1844

\section{Internet:}

www.nceas.usb.edu/ ^alroy/lefa/Loeb.html

www.brynmawr.edu/Acads/Psych/rwozniak/loeb.htm

Mahler, Gustav, österr. Komponist und Dirigent, ${ }^{*}$ Kalischt (Böhmen) 7. 7. 1860, † Wien 18. 5. 1911.

Lexika: Brockhaus 14, S. 48-49

\section{Biographie:}

Fischer, Jens Malte: Gustav Mahler : der fremde Vertraute; Biographie / Jens Malte Fischer. - Wien : Zsolnay, 2003. - 991 S. : Ill.

ISBN 3-552-05273-9

UB-Signatur: 2003 A 12800

Müller, Karl-Josef: Mahler : Leben - Werke - Dokumente / Karl-Josef Müller. - Mainz : Schott [u.a.], 1988. - 643 S. : zahlr. Ill., Notenbeisp. ISBN 3-492-18264-X

UB-Signatur: 88 A 12846

\section{Internet:}

de.wikipedia.org/wiki/Gustav_Mahler

Moltke, Helmuth Graf (1870) von, preuß. Generalfeldmarschall (1871), * Parchim 26. 10. 1800, † Berlin 24. 4. 1891.

\section{Lexika:}

Brockhaus 15, S. 25

Biographie: Kessel, Eberhard: Moltke. - Stuttgart : Koehler, 1957. 807 S. : Ill.

UB-Signatur: F 7125-17-61 


\section{Internet:}

de.wikipedia.org/wiki/Helmuth_Graf_von_Moltke

Mozart, Wolfgang Amadeus, Komponist, * Salzburg 27. 1. 1756, † Wien 5. 12. 1791.

\section{Lexika:}

Brockhaus 15, S. 180-182

\section{Biographie:}

Böttger, Dirk: Wolfgang Amadeus Mozart / Dirk Böttger. - München : Deutscher Taschenbuch-Verl., 2003. - 190 S. : Ill., Notenbeisp.

ISBN 3-423-31071-5

UB-Signatur: 2003 A 5468

Gutman, Robert W.: Mozart : a cultural biography / Robert W. Gutman. - New York [u.a.] : Harcourt + Brace, 1999. - XXII, 839 S. : Ill. ISBN 0-15-100482-X

UB-Signatur: 2000 A 6463

Schenk, Erich: Mozart : e. Biographie / Erich Schenk. - Mainz : Schott [u.a.], 1989. - 663 S. : Ill.

ISBN 3-7957-8268-6

UB-Signatur: FA 4479 MV

\section{Internet:}

de.wikipedia.org/wiki/Wolfgang_Amadeus_Mozart

www.mozartproject.org

Napoleon I., Kaiser der Franzosen (1804-14/15), urspr. Napoleon Buonaparte, ${ }^{*}$ Ajaccio (Korsika) 15. 8. 1769, $\dagger$ Longwood (Sankt Helena) 5. 5. 1821.

\section{Lexika:}

Brockhaus 15, S. 326-328

\section{Biographie:}

Englund, Steven: Napoleon : a political life / Steven Englund. - New York [u.a.] : Scribner, 2004. - XIV, 575 S. : Ill., Kt.

ISBN 0-684-87142-4

UB-Signatur: 2004 A 11175

Herre, Franz: Napoleon Bonaparte : Wegbereiter d. Jahrhunderts. München : Bertelsmann, 1989. - 415 S. : Ill.

ISBN 3-570-07569-9

UB-Signatur: 89 A 2716

\section{Internet:}

de.wikipedia.org/wiki/Napol\%C3\%A9on_Bonaparte 
Roosevelt Longworth, Alice, ${ }^{*}$ 12. 2. 1884, † 20. 2. 1980, Tochter der amerikan. Präsidenten Theodore Roosevelt.

\section{Internet:}

en.wikipedia.org/wiki/Alice_Roosevelt_Longworth www.nps.gov/sahi/kids.htm

Rowland, Henry Augustus, amerikan. Ingenieur und Physiker, ${ }^{*}$ Honesdale (Pa.) 27. 11. 1848, † Baltimore (Md.) 16.4. 1901; ab 1875 Prof. der Physik an der Johns Hopkins University. R. führte 1875/76 bei H. VON HELMHOLTZ grundlegende > Versuche über die elektromagnet. Wirkung elektr. Konvektion< durch; stellte die ersten reflektierenden Konkavgitter für Gitterspektrographen her und entwarf die erste Präzisionsmaschine zu deren Herstellung, 1899 erfand er einen Vierfachtypendrucker.

(aus Brockhaus)

\section{Lexika:}

Brockhaus 18, S. 579

\section{Biographie:}

Henry Rowland and astronomical spectroscopy : celebration of the 100. anniversary of Henry Rowland's Introduction of the concave diffraction grating ; proceedings of a symposium held in the Dep. of Physics and Astronomy, the Johns Hopkins Univ., Baltimore, 14 June 1984 / ed. by Richard C. Henry .... - Oxford [u.a.] : Pergamon Pr., 1986. - S. 115 - 355 : Ill., graph. Darst.

UB-Signatur: ZSN 5141 B::29,2

Schiller, (Johann Christoph) Friedrich von (seit 1802), Dichter, * Marbach am Neckar 10. 11. 1759, † Weimar 9. 5. 1805.

\section{Lexika:}

Brockhaus 19, S. 313-316

\section{Biographie:}

Pilling, Claudia: Friedrich Schiller / dargest. von Claudia Pilling, Diana Schilling u. Mirjam Springer. - Reinbek bei Hamburg : RowohltTaschenbuch-Verl., 2002. - 156 S. : zahlr. Ill.

ISBN 3-499-50600-9

UB-Signatur: 2002 A 3687

Wiese, Benno von: Friedrich Schiller / Benno von Wiese. - Stuttgart : Metzler, 1959. - XXI, 866 S.

UB-Signatur: G 6189-43 


\section{Internet:}

de.wikipedia.org/wiki/Friedrich_Schiller

Schubert, Franz (Peter), österr. Komponist, ${ }^{*}$ Lichtental (heute zu Wien) 31. 1. 1797, † Wien 19. 11. 1828.

\section{Lexika:}

Brockhaus 19, S. 477-479

\section{Biographie:}

Brown, Maurice J.: Schubert : eine kritische Biographie / Maurice J. E. Brown. - Wiesbaden : Breitkopf + Härtel, 1969. - XI, 417 S. : Notenbeisp.

UB-Signatur: 74 A 70

Gronau, Eduard: Franz Schubert : Musik zwischen Himmel und Abgrund ; eine Werkbiographie / Eduard Gronau. - Allensbach : Strehlow, 1993. - 512 S. : Ill.

ISBN 3-929735-00-8

UB-Signatur: 94 A 1595

Korff, Malte: Franz Schubert / von Malte Korff. - München : Dt. Taschenbuch-Verl., 2003. - 190 S. : Ill.

ISBN 3-423-31069-3

UB-Signatur: 2004 A 3956

\section{Internet:}

de.wikipedia.org/wiki/Franz_Schubert

Schweinfurth, Albert Cicero, ${ }^{*}$ Auburn 7. 1. 1864, $\dagger$ Dryden, NY 27. 9. 1900

also worked for Peabody \& Stearns from 1882 to 1885 and then in New York for A. Page Brown (1885-6). In the period 1886-90 he practised briefly with his eldest brother in Cleveland, worked alone and again with Brown in New York and then with the firm of Andrews and Jacques and indepently in Denver, CO; he rejoined Brown in San Francisco (1890-95) before establishing his own firm there (1895-8). Together with Brown he was ranked among the architects most instrumental in developing a regional Californian style based on a broad interpretation of Spanish and Spanish colonial precedents. His masterpieces in this style were the Hazienda dal Pozo de Verona (1895-6) in Pleasanton, CA und the Examiner Building (1897-8), San Francisco, both commissioned by William Randolph Hearst. His last major design, the First Unitarian Church (1898), Berkeley, CA, was an important structure in the newer, more abstract San Francisco Bay Area Tradition or Western Stick style, of which Bernard Maybeck became one of the chief 
exponents.

(aus Grove Dictionary)

\section{Lexika:}

The dictionary of art: [in thirty-four volumes] / ed. by Janes Turner.

- New York : Grove [u.a.]

Bd. 30, 1996. - S. 193-194

UB-Signatur: LSA Kunst-AA 005::30

\section{Biographie:}

Longstreth, Richard W.: On the edge of the world : four architects in San Francisco at the turn of the century. - New York : Architectural History Foundation, 1983. - XIV, 455 S. : Ill., Kt.

UB-Signatur: 84 A 2296

\section{Internet:}

www.berkelyheritage.com/berkeley_landmarks/1unitarian.htm www.vintagedesigns.com/id/latevic/ca/index.htm

Seydewitz, Paul von, * 1843, † 1910, sächsischer Kultusminister 1892-1906 Internet:

www.sachsen.de/de/ll/geschichte/regenten/1876-1918/inhalt_re.html

Sophokles, griech. Tragiker, ${ }^{*}$ Athen 497/496 v. Chr., † ebd. 406/405 v. Chr.

\section{Lexika:}

Brockhaus 20, S. 422-423

\section{Biographie:}

Flashar, Hellmut: Sophokles : Dichter im demokratischen Athen / Hellmut Flashar. - München : Beck, 2000. - 220 S.

ISBN 3-406-46639-7

UB-Signatur: LSA Alt-CA 011

\section{Internet:}

de.wikipedia.org/wiki/Sophokles

Stanford University, private Univ. in Palo Alto (Calif.), gegr. von dem Eisenbahnmagnaten und Senator LELAND STANFORD JR. (* 1824 , $†$ 1893), gegliedert in sieben große Fachbereiche. Die Hälfte der Studenten studiert ein halbes Jahr an Studienzentren der S. U. in Europa, Graduiertenstudium ist $\mathrm{u}$. a. in Japan und Taiwan möglich. Der Univ. angeschlossen sind u.a. das Dokumentationszentrum Hoover Institution on War, Revolution and Peace (gegr. 1919) und das Stanford Linear 
Accelerator Center (SLAC).

(aus Brockhaus)

Anm.: Der Gründer war Leland Stanford senior.

\section{Lexika:}

Brockhaus 21, S. 16

\section{Internet:}

www.stanford.edu/home/standford/history/begin.htm

Stanley, Albert A., 1851-1932, amerikan. Komponist

\section{Internet:}

www.umich.edu/ ${ }^{\sim}$ ummgc/conductors.htm

Steinway \& Sons, Unternehmen der Musikinstrumentenindustrie; gegr. 1853 in New York von dem 1850 in die USA ausgewanderten HEINRICH E. STEINWEG $(* 1797, \dagger 1871)$ und vier Söhnen. S. \& S. bauten erstmals 1855 ein Klavier mit einem in einem Stück gegossenen Eisenrahmen und mit einem kreuzsaitigen Bezug und wirkten durch zahlreiche Patente richtungsweisend im Klavierbau. Produktionsstätten befinden sich in New York und Hamburg. Gesellschafter: Steinway Musical Instruments, Inc., Boston (Mass.).

(aus Brockhaus)

\section{Lexika:}

Brockhaus 21, S. 79

\section{Biographie:}

Steinway + sons / Ronald V. Ratcliffe. Mit einem neuen Kapitel von Stuart Isacoff. [Aus d.Amerik. übers., bearb. u. erg. von Istvn N. Vrtes]. - Frankfurt am Main [u.a.] : Propyläen-Verl., 2002. - 227 S. : überw. Ill. ISBN 3-549-07192-2

UB-Signatur: 2003 A 2015

\section{Internet:}

de.wikipedia.org/wiki/Heinrich_Steinweg

Strauß, österr. Musikerfamilie des 19 und beginnenden 20. Jh., aus der mehrere der für die Wiener Tanzmusik und Operette maßgebl. Komponisten vorgegangen sind.

Bedeutende Vertreter:

1) Eduard, österr. Komponist, * Wien 15. 3. 1835, † ebd. 28.12. 1916, Sohn von 2); wirkte in der Kapelle seines Bruders JOHANN und wurde 1870 dessen Nachfolger als Hofballdirektor in Wien. ... Sein Sohn JOHANN STRAUSS $(* 1866, \dagger 1939)$ wirkte als sein Nachfolger zunächst 
in Wien, später als Kapellmeister in Berlin.

2) Johann (Vater), österr. Komponist * Wien 14. 3. 1804, † ebd. 25. 9. 1849, Vater von 1), 3) und 4); seit 1819 Bratschist in J. LANNERS Quartett, später in dessen Orchester Hilfsdirigent, gründete 1825 eine eigene Tanzkapelle. ... S. war neben LANNER der Begründer und der in ganz Europa gefeierte Repräsentant der Wiener Tanzmusik, bes. des Wiener Walzers. ...

3) Johann (Sohn), österr. Komponist, * Wien 25.10. 1825, † ebd. 3.6.1899, Sohn von 2), Bruder von l)und 4); gründete 1844 eine Tanzkapelle, die er nach dem Tod seines Vaters mit dessen Orchester vereinigte. Unter dem Beinamen > Walzerkönig < galt er auf vielen Konzertreisen bis in die USA als Repräsentant einer spezifisch wiener. Lebenshaltung und Musikkultur. ...

4) Josef, österr. Komponist, * Wien 22. 8. 1827, † ebd. 21. 7. 1870, Sohn von 2), Bruder von 1) und 3); war zunächst als Ingenieur tätig, widmete sich dann ebenfalls der Musik und vertrat häufig seinen Bruder JOHANN. ...

(aus Brockhaus)

\section{Lexika:}

Brockhaus 21, S. 243-244

\section{Biographie:}

Kemp, Peter: Die Familie Strauß : Geschichte e. Musikerdynastie / Peter Kemp. - Stuttgart : DVA, 1987. - 302 S. : Ill.

ISBN 3-421-06340-0

UB-Signatur: 87 A 4148

\section{Internet:}

de.wikipedia.org/wiki/Johann_Strau\%3C\%9F_\%28Vater\%29

de.wikipedia.org/wiki/Eduard_Strau\%3C\%9F

de.wikipedia.org/wiki/Josef_Strau\%3C\%9F

de.wikipedia.org/wiki/Johann_Strau\%3C\%9F_\%28Sohn\%29

Taft, William (Howard,) 27. Präs, der USA (1909-13), * Cincinnati (Oh.) 15. 9. 1857, † Washington (D.C.) 8. 3. 1930, Vater von 1) [Robert Alphonso Taft]; Jurist, gehörte als gemäßigt Konservativer der Republikan. Partei an; 1901-04 erster Zivil-Gouv. der Philippinen, ab 1904 Kriegs-Min. unter Präs. T. ROOSEVELT, gewann 1908 v. a. mit dessen Unterstützung die Präsidentschaftswahlen. Als Präs. initiierte er die Bekämpfung der großen Trusts und trat nachdrücklich für die Wahrung der amerikan Interessen in China und Mittelamerika ein (Dollardiplomatie). Er geriet zunehmend in Auseinandersetzung mit dem reformer. 
Flügel der Republikan. Partei, der sich 1912 unter Führung ROOSEVELTS als Progressive Party abspaltete. T. unterlag daraufhin bei den Präsidentschaftswahlen 1912 dem Demokraten T.W. WILSON. 191321 war T. Prof. für Rechtswiss. an der Yale University. Als Oberster Bundesrichter (1921-30) reformierte er das Supreme-Court-Verfahren und verfolgte in der Rechtsprechung einen konservativen, aber reformfreundl. Kurs.

(aus Brockhaus)

\section{Lexika:}

Brockhaus 21, S. 501

\section{Biographie:}

Burton, David H.: William Howard Taft : confident peacemaker / David H. Burton. - New York : Fordham Univ. Press, 2004. - VIII, 172 S. ISBN 0-916101-51-7

UB-Signatur: 2005 A 1693

Pringle, Henry F.: The life and times of William Howard Taft : a biography / by Henry F. Pringle. - New York [u.a.] : Farrar + Rinehart

Bd. 1. 1939. - XII, 555 S. : Ill.

Bd. 2. 1939. - VIII, S. 557-1106 : Ill.

Historisches Seminar

\section{Internet:}

en.wikipedia.org/wiki/William_Howard_Taft

Waldteufel, Emil, eigtl. Charles Émile Lévy, frz. Komponist, * Straßburg 9. 12. 1837, † Paris 16. 2. 1915; studierte am Pariser Conservatoire, wurde 1865 kaiserl. Hofballdirektor, dirigierte in Paris die Opernbälle, ferner in Berlin, Wien und London; komponierte über 250 Tänze für Orchester (v. a. Walzer), u. a. > Die Schlittschuhläufer $<$, $>$ Estudiantina $<$, $>$ Espana $<$, $>$ Sirenenzauber $<$, $>$ Mein Traum $<$, $>$ Dolores $<$.

(aus Brockhaus)

\section{Lexika:}

Brockhaus 23, S. 518

\section{Biographie:}

Fischbach, Bernard: La valse au coeur : Emile Waldteufel, le Strauss français / Bernard Fischbach ; Yves Waldteufel. - Strasbourg : Hirlé, 1997. - 135 S. : Ill., Notenbeisp.

ISBN 2-910048-51-9

UB-Signatur: 99 B 443

Lamb, Andrew: Skaters' Waltz : the story of the Waldteufels / by Andrew Lamb. - Croydon : Fullers Wood Press, 1995. - XII, 284 S. : Ill. 
ISBN 0-9524149-0-2

UB-Signatur: 97 A 234

\section{Internet:}

de.wikipedia.org/wiki/\%C3\%89mile_Waldteufel

Washington, George, amerikan. General und 1. Präs. der USA (1789-97),

* Wakefield (Westmoreland County, Va.) 22. 2. 1732, † Mount Vernon (Va.) 14. 12. 1799.

\section{Lexika:}

Brockhaus 23, S. 586-587

\section{Biographie:}

Göller, Josef-Thomas: George Washington : vom Waldläufer zum Staatsmann - der erste Präsident ; Biographie / Josef-Thomas Göller.

- Berlin : Edition q, 1998. - 392 S. : Ill., Kt.

ISBN 3-86124-355-5

UB-Signatur: 98 A 12087

Herre, Franz: George Washington : Präsident an der Wiege einer Weltmacht / Franz Herre. - Stuttgart : Dt. Verl.-Anst., 1999. - 415 S. : Ill. ISBN 3-421-05188-7

UB-Signatur: 99 A 8426

\section{Internet:}

de.wikipedia.org/wiki/George_Washington

Weierstraß, Karl (Theodor Wilhelm), Mathematiker, * Ostenfelde (heute zu Ennigerloh) 31.10. 1815, † Berlin 19. 2. 1897; 1856-64 Prof. am Gewerbeinstitut (heute TU) in Berlin, anschließend an der dortigen Universität. Neben B. RIEMANN, der den geometr. Standpunkt betonte, erarbeitete W. aus den Gesetzen der Potenzreihen eine Fundierung der Funktionentheorie. Er führte die Untersuchungen von N. H. ABEL fort, arbeitete über ellipt. Funktionen, analyt. Fortsetzung, die Produktdarstellung von Funktionen und über Singularitäten analyt. Funktionen. Auch zur Variationsrechnung leistete W. wichtige Beiträge. Seine Lehrtätigkeit übte nachhaltigen Einfluß auf die Entwicklung der Mathematik aus (>Berliner Schule $<$ ). Zu seinen Hörern zählten u. a. G. CANTOR, M. G. MITTAG-LEFFLER, SOFJA KOWALEWSKAJA und F. KLEIN.

(aus Brockhaus)

\section{Lexika:}

Brockhaus 23, S. 667

Meschkowski S. 263-266 
DMV Bd. 6, S. 7-44

ADB Bd. 55 S. 11-13

Pogg. II. Sp. 1282, III. S. 1424, IV. S. 1610

\section{Biographie:}

Festschrift zur Gedächtnisfeier für Karl Weierstrass : 1815-1965 / hrsg. von Heinrich Behnke .... - Köln [u.a.] : Westdt. Verl., 1966. - 612 S.

Bereichsbibliothek Mathematik

\section{Internet:}

www-history.mcs.st-and.ac.uk/history/Mathematicians/Weierstrass.html

Wheeler, Benjamin Ide, 1854-1927, Präsident der Berkeley University von 1899 bis 1919.

Internet:

www.infoplease.com/ce6/people/A0852034.html

Wilhelm II., Dt. Kaiser und König von Preußen (1888-1918), * Berlin 27. 1. $1859, \dagger$ Doorn 4. 6. 1941.

\section{Lexika:}

Brockhaus 24, S. 210-211

\section{Biographie:}

Herre, Franz: Kaiser Wilhelm II : Monarch zwischen den Zeiten / Franz Herre. - Köln : Kiepenheuer + Witsch, 1993. - 370 S. : Ill.

ISBN 3-462-02245-8

Wilhelm, Kronprinz des Dt. Reichs und von Preußen, * Potsdam 6. 5. 1882, $\dagger$ Hechingen 20. 7. 1951. Sohn aus erster Ehe von 3) [Wilhelm II.].

\section{Lexika:}

Brockhaus 24, S. 211

\section{Biographie:}

Herre, Paul: Kronprinz Wilhelm : seine Rolle in der deutschen Politik / Paul Herre. - München : Beck, 1954. - IX, 280 S. : Ill.

UB-Signatur: B 4439-40-40

Eppstein, Georg von: Der deutsche Kronprinz : Der Mensch, der Staatsmann, der Geschichtsschreiber / Georg Frhr. von Eppstein. - Leipzig : Koch, 1926. - 428 S., 38 Taf.

UB-Signatur: B 4439-40-30

Wirtinger, Wilhelm, ${ }^{*}$ Ybbs an der Donau 15. 7. 1865, † ebd. 15. 1. 1945, Mathematiker (Funktionentheorie). Von 1895-1903 Prof. in Innsbruck, dann bis 1935 Prof. in Wien. 


\section{Internet:}

www-history.mcs.st-andrews.ac.uk/Mathematicians/Wirtinger.html www.aeiou.at/aeiou.encyclop.w/w809593.htm

Zermelo, Ernst (Friedrich Ferdinand), Mathematiker, * Berlin 27. 7. 1871, $\dagger$ Freiburg im Breisgau 21.5. 1953; 1905 Titular-Prof. in Göttingen, ab 1910 Prof. in Zürich, 1916-26 wegen eines Lungenleidens als Privatgelehrter im Schwarzwald tätig, anschließend bis 1935 und ab 1946 Honorar-Prof. in Freiburg im Breisgau. Z. leistete Beiträge zur Variationsrechnung, Wahrscheinlichkeitsrechnung sowie Physik und wurde bes. durch zwei mit der expliziten Formulierung des Auswahlaxioms verbundene Beweise des Wohlordnungssatzes bekannt sowie durch seine bis 1908 entwickelte Axiomatisierung der Cantorschen Mengenlehre, die später von A. A. FRAENKEL und T. SKOLEM fortentwickelt wurde. Die axiomat. Mengenlehre förderte er mit weiteren Arbeiten und gab Werke von G. CANTOR heraus.

(aus Brockhaus)

\section{Lexika:}

Brockhaus 24, S. 544

\section{Internet:}

www-history.mcs.st-andrews.ac.uk/Mathematicians/Zermelo.html 


\section{Lexika und Biographiesammlungen}

\section{$\mathrm{ADB}$}

Allgemeine deutsche Biographie / hrsg. durch d. Histor. Comm. bei d. Königl. Akad. d. Wiss. - Leipzig [u.a.] : Duncker \& Humblot Info-Zentrum Altstadt IZA Biog-C-DE 006

Lesesaal Neuenheim LSN A-EH 002

\section{Brockhaus}

Brockhaus - die Enzyklopädie : in 24 Bänden. - 20. Aufl. - Leipzig ;

Mannheim : Brockhaus

ISBN 3-7653-3100-7

Lesesaal Altstadt LSA Al-A-DE 010

Lesesaal Neuenheim LSN A-BE 001

\section{DBE}

Deutsche biographische Enzyklopädie / hrsg. von Walther Killy ... -

München [u.a.] : Saur

ISBN 3-598-23186-1

Info-Zentrum Altstadt IZA Biog-C-DE 022

\section{DMV}

Jahresbericht der Deutschen Mathematiker-Vereinigung. - Stuttgart [u.a.] : Teubner

$$
\begin{array}{ll}
\text { Magazin Landfriedhaus } & \text { L } 22 \text { (1890-1962) } \\
\text { Magazin Neuenheim } & \text { ZSN } 2000 \text { B (1962-1994) }
\end{array}
$$

\section{DSB}

Dictionary of scientific biography / Charles C. Gillispie, ed. in chief ;

American Council of Learned Societies. - New York : Scribner

$\begin{array}{lll}\text { Info-Zentrum Altstadt } & \text { IZA Biog-C } & 017 \\ \text { Lesesaal Neuenheim } & \text { LSN B-AH } & 014\end{array}$

\section{HGL}

Heidelberger Gelehrtenlexikon : 1803 - 1932 / Dagmar Drll. - Berlin [u.a.], 1996

$\begin{array}{ll}\text { Info-Zentrum Altstadt } & \text { IZA Biog-D-HEI } 002 \\ \text { Lesesaal Neuenheim } & \text { A-BE } 038\end{array}$

Meyer

Meyers enzyklopädisches Lexikon : in 25 Bänden. - 9. Aufl. - Mannheim [u.a.] : Bibliogr. Inst.

Lesesaal Altstadt LSA Al-A-DE 004

Lesesaal Neuenheim LSN A-BE 002 


\section{Meschkowski}

Mathematiker-Lexikon / von Herbert Meschkowski. - Mannheim [u.a.]

: Bibliogr. Inst., 1964

Lesesaal Neuenheim LSN D-BA 026

\section{NDB}

Neue deutsche Biographie / hrsg. von der Historischen Kommission bei der Bayerischen Akademie der Wissenschaften. - Berlin : Duncker \& Humblot

ISBN 3-428-00181-8

Info-Zentrum Altstadt IZA Biog-C-DE 007

Lesesaal Neuenheim LSN A-EH 001

\section{Pagel}

Biographisches Lexikon hervorragender Ärzte des neunzehnten Jahrhunderts : mit einer historischen Einleitung / hrsg. von J. Pagel. Berlin ; Wien : Urban \& Schwarzenberg, 1901

Landfriedhaus P 214-58

\section{Pogg}

Poggendorff, Johann C.: Biographisch-literarisches Handwörterbuch zur Geschichte der exacten Wissenschaften. - Leipzig : Barth

Bd. 1. 1863 - Bd. 7. 1973

Lesesaal Altstadt LSA Nat-A 001

Lesesaal Neuenheim LSN B-AE 002 